\title{
A Study of the 20 day Superorbital Modulation in the High-mass X-Ray Binary IGR J16493-4348
}

\author{
Joel B. Coley ${ }^{1,2}$ (1), Robin H. D. Corbet ${ }^{3,4}$ (1) Felix Fürst ${ }^{5}$ (D), Gregory Huxtable ${ }^{3,4}$, Hans A. Krimm ${ }^{6,7}$ (1), \\ Aaron B. Pearlman ${ }^{8,9,10}$ (iD), and Katja Pottschmidt ${ }^{2,3}$ (iD \\ ${ }^{1}$ Department of Physics and Astronomy, Howard University, Washington, DC 20059, USA; joel.coley@ howard.edu \\ ${ }^{2}$ CRESST/Mail Code 661, Astroparticle Physics Laboratory, NASA Goddard Space Flight Center, Greenbelt, MD 20771, USA \\ ${ }^{3}$ University of Maryland, Baltimore County, MD, USA \\ ${ }^{4}$ CRESST/Mail Code 662, X-ray Astrophysics Laboratory, NASA Goddard Space Flight Center, Greenbelt, MD 20771, USA \\ ${ }^{5}$ European Space Astronomy Center (ESA/ESAC), Operations Department, Vilanueva de la Cañada (Madrid), Spain \\ ${ }^{6}$ Universities Space Research Association, Columbia, MD 21046, USA \\ ${ }^{7}$ National Science Foundation, Alexandria, VA 22314, USA \\ ${ }^{8}$ Division of Physics, Mathematics, and Astronomy, California Institute of Technology, Pasadena, CA 91125, USA \\ Received 2018 October 9; revised 2019 May 3; accepted 2019 May 15; published 2019 July 1
}

\begin{abstract}
We report on Nuclear Spectroscopic Telescope Array (NuSTAR), Neil Gehrels Swift Observatory (Swift) X-ray Telescope (XRT), and Swift Burst Alert Telescope (BAT) observations of IGR J16493-4348, a wind-fed supergiant $\mathrm{X}$-ray binary showing significant superorbital variability. From a discrete Fourier transform of the BAT light curve, we refine its superorbital period to be $20.058 \pm 0.007$ days. The BAT dynamic power spectrum and a fractional root mean square analysis both show strong variations in the amplitude of the superorbital modulation, but no observed changes in the period are found. The superorbital modulation is significantly weaker between MJD 55,700 and MJD 56,300. The joint NuSTAR and XRT observations, which were performed near the minimum and maximum of one cycle of the 20 day superorbital modulation, show that the flux increases by more than a factor of two between superorbital minimum and maximum. We find no significant changes in the 3-50 keV pulse profiles between superorbital minimum and maximum, which suggests a similar accretion regime. Modeling the pulse-phase-averaged spectra we find a possible $\mathrm{Fe} \mathrm{K} \alpha$ emission line at $6.4 \mathrm{keV}$ at superorbital maximum. This feature is not significant at superorbital minimum. While we do not observe any significant differences between the pulse-phase-averaged spectral continua apart from the overall flux change, we find that the hardness ratio near the broad main peak of the pulse profile increases from superorbital minimum to maximum. This suggests the spectral shape hardens with increasing luminosity. We discuss different mechanisms that might drive the observed superorbital modulation.
\end{abstract}

Key words: accretion, accretion disks - stars: individual (IGR J16493-4348) - stars: neutron - X-rays: binaries

\section{Introduction}

IGR J16493-4348 is a high-mass X-ray binary first discovered during a survey of the Galactic plane using the INTErnational Gamma-Ray Astrophysics Laboratory (INTEGRAL; Winkler et al. 2003) satellite (Bird et al. 2004). During a deep scan of the Norma Arm region using INTEGRAL, it was later identified by Grebenev et al. (2005) to be a variable source with a mean photon flux of $5.6 \pm 0.6 \mathrm{mCrab}$ in the $18-45 \mathrm{keV}$ energy band. Two pointed observations using the Rossi X-ray Timing Explorer (RXTE) Proportional Counter Array (PCA) revealed the mean X-ray spectrum to be consistent with a highly absorbed power law. Its photon index and neutral hydrogen absorbing column were found to be 1.4 and $\sim 10^{23} \mathrm{~cm}^{-2}$, respectively (Markwardt et al. 2005).

A spectral analysis using the Neil Gehrels Swift Observatory (Swift) Burst Alert Telescope (BAT) and the INTEGRAL Soft Gamma-ray Imager (ISGRI), together with pointed Swift X-ray Telescope (XRT) and Suzaku observations, revealed a hint of an absorption feature at $33 \pm 4 \mathrm{keV}$ thought to be a cyclotron resonant scattering feature (CRSF), implying a magnetic field of $(3.7 \pm 0.4) \times 10^{12} \mathrm{G}$ (D'Aì et al. 2011). The width of the absorption feature was found to be $10 \mathrm{keV}$.

\footnotetext{
9 NDSEG Research Fellow.

${ }^{10}$ NSF Graduate Research Fellow.
}

A single source in the Two Micron All-Sky Survey (2MASS) catalog, 2MASS J16492695-4349090, was identified as the infrared counterpart (Kuiper et al. 2005). Using the European Southern Observatory Infrared Spectrometer and Array Camera spectrograph on UT1 at Paranal observatory, Nespoli et al. (2010) proposed the spectral type of the donor star to be B0.5-1 Ia-Ib. The distance to the source was estimated to be between 6 and $26 \mathrm{kpc}$ by Nespoli et al., but could not be tightly constrained due to the uncertainty of the intrinsic colors.

A 6.78 day orbital period was independently found by Corbet et al. (2010a) and Cusumano et al. (2010). It was later refined by Corbet \& Krimm (2013) to be $6.782 \pm 0.001$ days using the BAT Transient Monitor. The neutron star is regularly eclipsed by the donor star for about 0.8 days of every orbit, which indicates the orbital inclination is close to edge-on (Pearlman et al. 2019). From an eclipse timing analysis using the Swift BAT and RXTE PCA, Pearlman et al. (2019) further refined the orbital period to be $6.7828 \pm 0.0004$ days.

Recently, Pearlman et al. (2019) placed constraints on the nature of the donor star using their eclipse timing results. They proposed the spectral type of the donor star and the distance to the source to be B0.5 Ia and $16.1 \pm 1.5 \mathrm{kpc}$, respectively. We adopt these measurements in this work.

Corbet et al. (2010b) found evidence of a $\sim 1069$ s signal using the RXTE PCA, which they interpreted as the neutron star 
rotation period. From a pulsar timing analysis using an extended PCA data set, this was later refined to $1093.1036 \pm 0.0004 \mathrm{~s}$ (Pearlman et al. 2019). The epoch of maximum delay time, $T_{\pi / 2}$, and pulse period derivative were found to be MJD 55,850.91 \pm 0.05 and 5.4 $-9.7 \times 10^{-8} \mathrm{~s} \mathrm{~s}^{-1}$, respectively (Pearlman et al. 2019).

In addition to the neutron star rotation and orbital periods, a longer superorbital period was observed from IGR J164934348. Using data from the Swift BAT 58 month survey and the RXTE Galactic plane scans, a $\sim 20$ day modulation was found (Corbet et al. 2010a). The superorbital period was later refined to be $20.07 \pm 0.01$ days using the BAT Transient Monitor (Corbet \& Krimm 2013). More recently, Pearlman et al. (2019) refined the superorbital period to be $20.067 \pm 0.009$ days also using the BAT.

Superorbital modulation was additionally seen in the windfed supergiant X-ray binaries (SGXBs) 2S 0114+650 (Farrell et al. 2008), IGR J16418-4532, IGR J16479-4514, and 4U 1909+07 (Corbet \& Krimm 2013). More recently, Corbet et al. (2018) reported evidence of superorbital modulation in $4 \mathrm{U}$ 1538-522. In their review of wind-fed SGXBs showing strong superorbital modulation, Corbet \& Krimm (2013) found a possible correlation between the orbital and superorbital periods of these binaries, but the mechanism to account for this correlation remains unclear. Superorbital variability in wind-fed SGXBs is not a ubiquitous feature since many windfed SGXBs show strong orbital modulation but no signs of superorbital modulation (Corbet \& Krimm 2013).

In Roche-lobe overflow systems, superorbital variations can typically be explained by X-ray irradiation from a central source illuminating a tilted and/or warped accretion disk, causing it to precess and periodically obscure the compact object from the line of sight (Pringle 1996; Ogilvie \& Dubus 2001). Similar variability has also recently been found in ultraluminous X-ray (ULX) pulsars (e.g., NGC 5907 ULX1; NGC 7793 P13; M82 X-2, Walton et al. 2016; Fürst et al. 2018; Brightman et al. 2019). However, the mechanism responsible for the long-timescale modulation in wind-fed SGXBs remains poorly understood. Depending on the angular momentum transferred to the compact object by the stellar wind, accretion in wind-fed SGXBs may be mediated by a quasi-spherical outflow (Bondi \& Hoyle 1944) or by an accretion disk-like structure albeit of a transient nature (El Mellah et al. 2019; Taani et al. 2018, 2019). Indeed, transient accretion disks have been observed in some wind-fed SGXBs (e.g., OAO 1657-415, 2S 0114+650; Jenke et al. 2012; Hu et al. 2017). While it is unlikely that a precessing warped and/or tilted accretion disk is the primary mechanism that drives superorbital modulation in wind-fed SGXBs, it is possible that the superorbital variations are caused by a variable mass accretion rate. Possible mechanisms that could drive superorbital variations in wind-fed SGXBs include neutron star precession (Postnov et al. 2013), donor star variability (Koenigsberger et al. 2006), or the presence of a third star in a hierarchical system (Chou \& Grindlay 2001). Recently, Bozzo et al. (2017) proposed that a corotation interaction region with a period of $\sim 10.3$ days could explain the $\sim 20.07$ day superorbital period and amplitude in IGR J16493-4348.

In this paper, we analyze two Nuclear Spectroscopic Telescope Array (NuSTAR) and Swift XRT observations of IGR J16493-4348 near the maximum and the minimum of one cycle of the $\sim 20$ day superorbital modulation, together with
Swift BAT Transient Monitor observations, which track the evolution of the superorbital modulation on long timescales. The remainder of the paper is organized as follows. NuSTAR and Swift observations are presented in Section 2. Section 3.1 focuses on long-term monitoring of the $\sim 20$ day superorbital modulation with the Swift BAT. In Section 3.2, we measure the neutron star rotation period using the NuSTAR X-ray telescope and show pulse profiles and their energy dependence at superorbital minimum and superorbital maximum. Pulsephase-averaged and phase-resolved spectral results are given in Sections 3.3 and 3.4, respectively. Section 3.5 focuses on the spectroscopy at the peak of the pulse profile. We provide a discussion of the results in Section 4 and the conclusions are given in Section 5. Unless stated otherwise, the uncertainties and limits presented in the paper are at the $90 \%$ confidence level.

\section{Data and Analysis}

The observations outlined below consist of nearly simultaneous NuSTAR and Swift XRT observations during superorbital minimum (2015 August 31-September 1) and superorbital maximum (2015 September 12), as well as long-term observations of the system with the Swift BAT. An observation $\log$ is given in Table 1 .

\subsection{NuSTAR Observations}

NuSTAR (Harrison et al. 2013) carries two co-aligned grazing incidence Wolter I imaging telescopes that focus $\mathrm{X}$-rays between 3 and $79 \mathrm{keV}$ onto two independent solid state Focal Plane Modules (hereafter FPMA and FPMB). We reduced and screened the data using the NuSTAR Data Analysis Software (NuSTARDAS) v.1.7.0 package provided under HEAsoft v.6.20 and calibration files dated 2016 December 7. The data were reprocessed with the NuSTARDAS data pipeline package nupipeline using the standard filtering procedure to apply the newest calibration and default screening criteria.

The source spectra were extracted in mode 01 (SCIENCE) from a circular region of radius 60 " 0 centered on the source. Since the Norma Arm is a crowded region, we checked for stray light contamination produced by sources outside the field of view using the scripts made available on the NuSTAR GitHub webpage. ${ }^{11}$ We found that FPMA is affected by stray light from multiple sources. To investigate variations in the background due to stray light, we tested different background regions on the same detector as the source while avoiding visible stray light. We found the spectral parameters do not significantly depend on the choice of background (see Sections 3.3-3.5). This is not surprising since IGR J164934348 was found to be a factor of 10 times brighter than the background at energies below $30 \mathrm{keV}$ and a factor of two at energies above $30 \mathrm{keV}$. We therefore chose to extract a background from a circular region of radius 60 ". 0 offset from the source. Event times were corrected to the solar system barycenter using nuproducts and the FTOOL barycorr with the DE-200 solar system ephemeris. For the timing analysis and pulse-phase-resolved spectra, we further corrected the event times for the orbital motion of the neutron star using the ephemeris defined in Pearlman et al. (2019), which

\footnotetext{
11 https://github.com/NuSTAR
} 
Table 1

Summary of X-Ray Observations of IGR J16493-4348

\begin{tabular}{|c|c|c|c|c|c|c|c|}
\hline Obs. & Telescope & ObsID & $\begin{array}{l}\text { Start Time } \\
\text { (UT) }\end{array}$ & $\begin{array}{l}\text { End Time } \\
\text { (UT) }\end{array}$ & Orbital Phase $^{\mathrm{a}}$ & Superorbital Phase ${ }^{\mathrm{b}}$ & $\begin{array}{c}\text { Exposure } \\
(\mathrm{ks})\end{array}$ \\
\hline Min & NuSTAR & 30102054004 & 2015 Aug 31 07:23:41 & 2015 Sep 01 00:26:38 & $0.527-0.632$ & $0.520-0.555$ & 31.2 \\
\hline Max & NUSTAR & 30102054006 & 2015 Sep 12 04:40:20 & 2015 Sep 12 15:17:55 & $0.280-0.344$ & $1.113-1.135$ & 21.6 \\
\hline Min & Swift & 00081665002 & 2015 Aug 31 10:25:51 & 2015 Aug 31 10:34:38 & $0.545-0.546$ & $0.526-0.527$ & $0.5^{\mathrm{c}}$ \\
\hline Max & Swift & 00081665003 & 2015 Sep 12 13:00:01 & 2015 Sep 12 14:56:54 & $0.331-0.343$ & $1.130-1.134$ & $1.9^{\mathrm{c}}$ \\
\hline
\end{tabular}

Notes.

a Orbital phase zero is defined at MJD 55,851.2, corresponding to the epoch of maximum delay time, $T_{\pi / 2}$ (Pearlman et al. 2019).

b Superorbital phase zero is defined as the epoch of maximum flux (MJD 57,254.9 \pm 0.3 ).

${ }^{\mathrm{c}}$ Net exposure time is spread over several snapshots.

assumed a circular orbital solution and no change in the neutron star rotation period (see Section 1). For phase-resolved spectra, good time intervals were generated using the nuproducts tool and the "usrgtifile" keyword. Response matrices were generated using the packages numkarf and numkrmf.

The net count rates from the source over the full energy range were found to be $0.595 \pm 0.004$ counts $^{-1}$ (FPMA) and $0.571 \pm 0.004$ counts $\mathrm{s}^{-1}$ (FPMB) at superorbital minimum and $1.679 \pm 0.009$ counts $\mathrm{s}^{-1}$ (FPMA) and $1.651 \pm 0.009$ counts $\mathrm{s}^{-1}$ (FPMB) at superorbital maximum. The background was found to dominate at energies exceeding $\sim 40 \mathrm{keV}$ at superorbital minimum and $\sim 50 \mathrm{keV}$ at superorbital maximum. As a result, we chose to analyze the spectra between $3-40 \mathrm{keV}$. We rebinned the spectral file produced by nuproducts to have a minimum of 50 counts per bin using grppha.

\subsection{Swift}

\subsubsection{XRT Observations}

The Swift XRT (Burrows et al. 2005) is a Wolter I imaging telescope sensitive to X-rays ranging from 0.3 to $10 \mathrm{keV}$. We reduced and screened the data using the HEAsoft v.6.20 package and calibration files dated 2017 May 1, following the procedures defined in the XRT Data Reduction Guide (Capalbi et al. 2005). The data were reprocessed with the XRTDAS standard data pipeline package xrtpipeline using the standard filtering procedure to apply the newest calibration and default screening criteria. All data were taken in photon counting (PC; Hill et al. 2004) mode with a data readout time of $2.5 \mathrm{~s}$, adopting the standard grade filtering (0-12 for PC).

We found the non-background-subtracted count rates at superorbital minimum and superorbital maximum to be $0.15 \pm 0.01$ counts $\mathrm{s}^{-1}$ and $0.25 \pm 0.01$ counts $\mathrm{s}^{-1}$, respectively. Since our observations of IGR J16493-4348 were not affected by pile-up, we extracted the source spectra from circular regions of radius $30^{\prime \prime}$ centered on the source. The backgrounds were extracted from an annular region of internal radius $60^{\prime \prime}$ and external radius $120^{\prime \prime}$ centered on the source. The ancillary response files, accounting for vignetting, point-spread function correction, and different extraction regions, were generated and corrected for exposure using the FTOOL packages xrtmkarf and xrtexpomap, respectively.

We further processed the spectral data produced by xselect using the FTOOL grppha, which defined the binning and quality flags of the spectra. We used the quality flag to further eliminate bad data. Bins were grouped to ensure a minimum of 20 counts per bin.

\subsubsection{BAT Observations}

The BAT, on board the Swift spacecraft, is a hard X-ray telescope operating in the 14-195 keV energy band (Barthelmy et al. 2005). It provides an all-sky hard X-ray survey with a sensitivity of $\sim 1 \mathrm{mCrab}$ (Tueller et al. 2010). We analyzed BAT data obtained during the time period MJD 53,416-57,923 (2005 February 15-2017 June 19). Light curves were retrieved using the extraction of the BAT Transient Monitor data available on the NASA GSFC HEASARC website ${ }^{12}$ (Krimm et al. 2013). We used the orbital light curves in the $15-50 \mathrm{keV}$ energy band in our analysis, which have exposures that range from 64 to $2640 \mathrm{~s}$ in each time bin (see Section 3.1). The mean exposure in the time bins was $706 \mathrm{~s}$. The short exposures can arise due to the observing plan of Swift since the BAT is primarily tasked to observe gamma-ray bursts (Krimm et al. 2013).

The light curves were further screened to exclude bad quality points. We only considered data where the data quality flag ("DATA_FLAG") was set to 0, indicating good quality. Data flagged as "good" are sometimes suspect, where a small number of data points with very low fluxes and implausibly small uncertainties are found (Corbet \& Krimm 2013). These points were removed from the light curves. We corrected the photon arrival times to the solar system barycenter using the scripts made available on the Ohio State Astronomy webpage. ${ }^{13}$

\section{Results \\ 3.1. Long-term Variability}

The Swift BAT Transient Monitor light curve of IGR $\mathrm{J} 16493-4348$ is shown in Figure 1. We rebinned the light curve to two superorbital cycles and found no major variability over the duration of the light curve.

We derived the superorbital period using a discrete Fourier transform (DFT) of the BAT light curve, after removing points that fell within the eclipse of the neutron star from the start of ingress to the end of egress using the ephemeris defined in Pearlman et al. (2019). The DFT covered a period range between 0.07 days and the length of the light curve-i.e., $\sim 4507$ days. We weighted the contribution of each data point

\footnotetext{
12 http://heasarc.gsfc.nasa.gov/docs/swift/results/transients/

13 http://astroutils.astronomy.ohio-state.edu/time/
} 


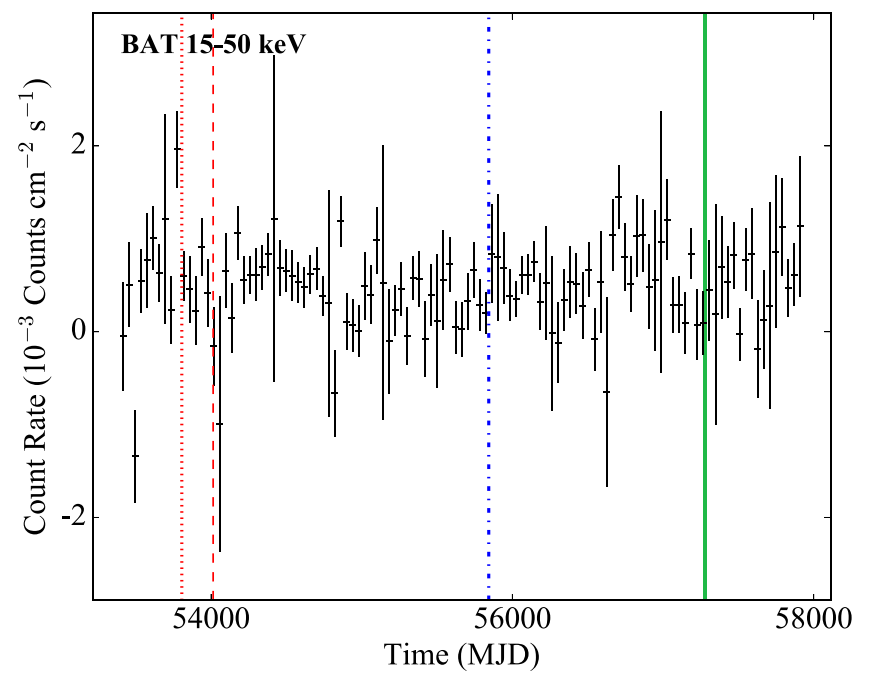

Figure 1. Long-term Swift BAT light curve of IGR J16493-4348 in the 15-50 keV band (MJD 53,416-57,923). The light curve is binned to a time resolution chosen to be two superorbital cycles ( $\sim 40.13$ days). The superorbital cycle coinciding with the times of the NUSTAR observations is indicated by the green shaded region. The Swift XRT, Suzaku, and RXTE PCA observations reported in Hill et al. (2008), Morris et al. (2009), and Pearlman et al. (2019), respectively, are indicated by the dotted red, dashed red, and dotted-dashed blue lines, respectively.

by its uncertainty when calculating the power spectrum using the "semi-weighting" technique (Corbet et al. 2007; Corbet \& Krimm 2013), where the error bars on each data point and the excess variability of the light curve are taken into account. The significance of the peak at the superorbital period was estimated using the false-alarm probability (FAP; Scargle 1982), which depends on the number of independent frequencies and therefore the nominal frequency resolution. While this is not precisely defined for unevenly sampled data (Koen 1990), the inverse of the light-curve length provides a reasonable approximation (Corbet et al. 2017). The uncertainty in our period measurements is obtained using the expression given in Horne \& Baliunas (1986).

The $\sim 20$ day superorbital modulation is strongly detected in the DFT of the BAT Transient Monitor light curve. Using an additional 674 days of data compared to Pearlman et al. (2019), we refine the superorbital period to $20.058 \pm 0.007$ days (see Figure 2(c)). The FAP is $3 \times 10^{-7}$. We note that by excising the eclipses, gaps with a spacing of about 1.7 days are created in the light curve, which could possibly lead to aliasing effects in the power spectrum. To investigate this, we created a light curve using the times of the BAT light and replaced the data values with a sinusoidal modulation at 20.058 days. We found no evidence of aliasing in the power spectrum.

To monitor changes in the $\sim 20.06$ day modulation, we constructed dynamic power spectra using the Swift BAT light curve (see Figure 2(a)). To investigate whether changes in the signal were sudden or gradual, overlapping light curve subsets were used (e.g., Clarkson et al. 2003). We divided the light curve into 70 data windows, each with a length of 1000 days, that were successively shifted in time by 50 days relative to each other. We calculated the DFT from each subset of data. In Figure 2(a), we show that the amplitude of the $\sim 20.06$ day modulation changes as a function of time. We find no change in the period of the $\sim 20.06$ day modulation.

In Figure 2(b), we show changes in the strength of the $\sim 20.06$ day modulation relative to the average value for each of the individual 70 power spectra. We find the peak power to be more than 10 times the mean power up to $\sim$ MJD 54,300 and again at MJD 55,000-55,200 (2009 September 26-2010 January 4). The relative peak height is found to be near constant at five times the mean power level between MJD 54,300-55,000. From MJD 55,500 to MJD 56,100 (2012 June 22), we find that it decreases, reaching a minimum of 1.3 times the mean power level. The power then increases linearly up to MJD 56,400 (2013 April 18) where it is again larger than five times the mean power level.

Quasi-sinusoidal behavior was found in the BAT Transient Monitor light curve folded on the superorbital period (see Figure 3(c)) where we defined phase zero as the epoch of the maximum flux derived from a sine wave fit. Since the NuSTAR observation near superorbital minimum began at MJD 57,265.3 (see Table 1), we express the epoch of maximum flux (MJD 57,254.9 \pm 0.3 ) at an epoch closest to the NuSTAR observation assuming no appreciable change in the superorbital period.

We investigated changes in the amplitude and phase of the superorbital modulation using a dynamic folded light curve (see Figure 3(a)). We divided the light curve into 70 data windows, which each had a length of 1000 days and were shifted in time by 50 days relative to each other. We folded the light curve from each subset of data on the $20.058 \pm 0.007$ day period. The dynamic folded light curve shows a maximum and minimum near superorbital phases $\sim 0.9-0.2$ and $\sim 0.4-0.6$, respectively.

To further investigate changes in the amplitude of the modulation, we calculated the fractional root mean square (rms) amplitude and its uncertainty for each 1000 day segment using Equations (10) and (B2) in Vaughan et al. (2003), respectively (see Figure $3(\mathrm{~b})$ ). We find the fractional rms amplitude to track the power of the $\sim 20.06$ day modulation as a function of time. The fractional rms analysis shows the amplitude of the superorbital modulation significantly decreased to less than 3\% between MJD 55,700 and MJD 56,300, which is consistent with the weakening in the dynamic power spectrum. The weighted Pearson correlation coefficient between the fractional $\mathrm{rms}$ and relative height is found to be $r=0.83$, with a probability of arising by chance of $5 \times 10^{-8}$.

\subsection{Short-term Temporal Analysis}

In Figure 4(a), we show the Swift BAT light curve folded on the $20.058 \pm 0.007$ day superorbital period using the ephemeris defined in Section 3.1 along with the FPMA light curves, binned to a resolution of $500 \mathrm{~s}$. This illustrates that the NuSTAR observations coincide with superorbital minimum and maximum.

Using the ephemeris defined in Pearlman et al. (2019), we also folded the Swift BAT light curve on the $6.7828 \pm$ 0.0004 day orbital period (see Figure 4(b)). The NuSTAR FPMA superorbital minimum and maximum light curves are overplotted in red. This shows that the NuSTAR observations are clearly outside of eclipse.

To determine the neutron star rotation period in both NUSTAR observations, we used the epoch folding technique presented in Leahy (1987) applied to the combined FPMA + FPMB light curves binned to a resolution of $1 \mathrm{~s}$. We estimated the uncertainty on the pulse period at the $1 \sigma$ confidence interval by simulating 2000 light curves based on 


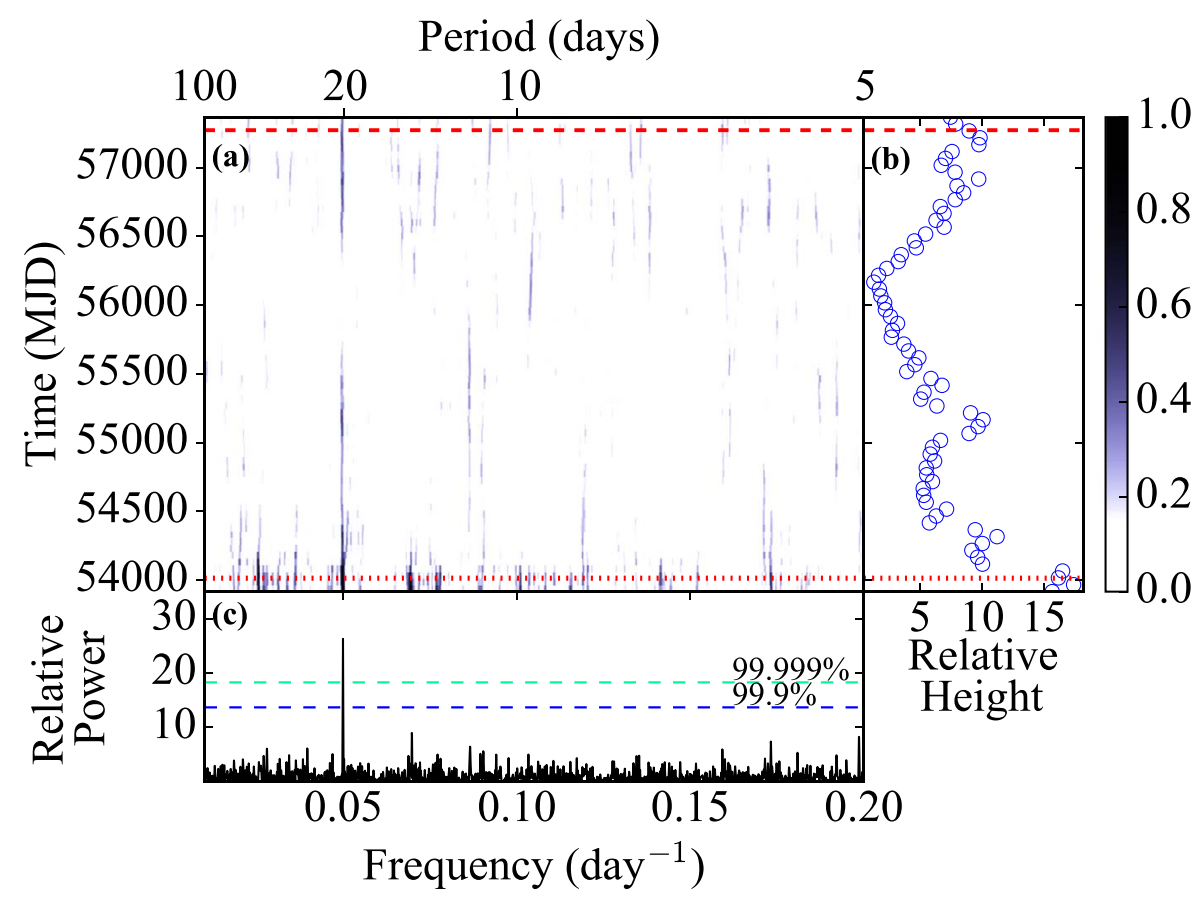

Figure 2. (a) Swift BAT dynamic power spectrum in the $15-50 \mathrm{keV}$ band of IGR J16493-4348 as a function of time. The power spectra were calculated using 1000 day time intervals, with 50 day increments in the start and end times. The superorbital cycle coinciding with the times of the NuSTAR observations near superorbital minimum and maximum is indicated by the red dashed line. The Suzaku observation reported in Morris et al. (2009) is indicated by the dotted red line. (b) Relative height of the peak near the $\sim 20.06$ day superorbital period in the power spectrum for each 1000 day time interval. (c) Discrete Fourier transform of the entire data set produced, with $99.9 \%$ and $99.999 \%$ significance levels indicated by the blue and green dashed lines, respectively.

the previously determined pulse period and profile with additional Poisson noise. We find the neutron star rotation period at superorbital minimum and maximum to be $1092.9 \pm$ $0.2 \mathrm{~s}$ and $1092.6 \pm 0.3 \mathrm{~s}$, after correcting for the binary orbital motion. The neutron star rotation period shows no significant change between superorbital minimum and maximum. To investigate changes in the neutron star rotation period between our NUSTAR observations and the spin period reported in Pearlman et al. (2019), we calculated the Taylor expansion (see Equation (1)),

$$
P(t)=P\left(t_{0}\right)+\left(t-t_{0}\right) \dot{P}
$$

where $P(t)$ is the neutron star rotation period derived at superorbital minimum, $P\left(t_{0}\right)$ is the neutron star rotation period derived in Pearlman et al. (2019), and the epoch $t_{0}$ is the maximum delay time from the pulsar timing analysis (Pearlman et al. 2019). We find the pulse period derivative between the RXTE observations reported in Pearlman et al. and our NUSTAR observation at superorbital minimum to be $(-1.8 \pm 1.7) \times$ $10^{-9} \mathrm{~s} \mathrm{~s}^{-1}$, which is consistent with zero.

In Figure 5(a), we show the NUSTAR FPMA light curves near superorbital minimum and maximum binned to a time resolution chosen to be the mean pulse period $(\sim 1092.7 \mathrm{~s})$. We divided the light curves into two energy bands, where the soft band is defined between energies $3-10 \mathrm{keV}$ and characterized by the count rate $C_{\text {soft }}$, and the hard band is between $15-50 \mathrm{keV}$ and denoted by the count rate $C_{\text {hard }}$. We define the hardness ratios as

$$
\mathrm{HR}=\frac{\left(C_{\text {hard }}-C_{\text {soft }}\right)}{\left(C_{\text {hard }}+C_{\text {soft }}\right)},
$$

where a soft spectrum is indicated by negative values and a hard spectrum is indicated by positive values (see Figure 5(b)).
While the X-ray flux significantly increases between superorbital minimum and maximum, no change in the hardness ratio between superorbital minimum and maximum was found.

We folded the NUSTAR 3-50 keV light curves at superorbital minimum and maximum on the $\sim 1092.9 \mathrm{~s}$ and $\sim 1092.6 \mathrm{~s}$ periods, respectively. For the observation at superorbital maximum, we defined phase zero at the time of maximum delay (see Section 1). We aligned the pulse profiles at superorbital minimum and maximum by calculating the maximum value of the cross-correlation function between the two pulse profiles (see Figure 6). The pulse profiles at superorbital minimum and maximum each show a doublepeaked structure with a main broad peak and a smaller secondary peak.

To investigate the energy dependence of the pulse profile, we divided the light curve into five energy bands defined between energies of $3-6 \mathrm{keV}, 6-10 \mathrm{keV}, 10-20 \mathrm{keV}, 20-30 \mathrm{keV}$, and $30-50 \mathrm{keV}$. The pulse profiles at both superorbital minimum and maximum show a weak energy dependence (see Figure 6). Only small changes in the pulse profiles are seen, where the main peak is broad up to $20 \mathrm{keV}$ and progressively becomes narrower up to $\sim 50 \mathrm{keV}$ (see Figures 6(b)-(f)).

We define the peak-to-peak pulse fraction as

$$
\mathcal{P}=\frac{\left(F_{\max }-F_{\min }\right)}{\left(F_{\max }+F_{\min }\right)}
$$

where the maximum and minimum count rates in the pulse profile are characterized as $F_{\max }$ and $F_{\min }$, respectively. Using Equation (3), we found that the peak-to-peak pulsed fraction at both superorbital minimum and maximum increases with increasing energy (see Figure 7). 


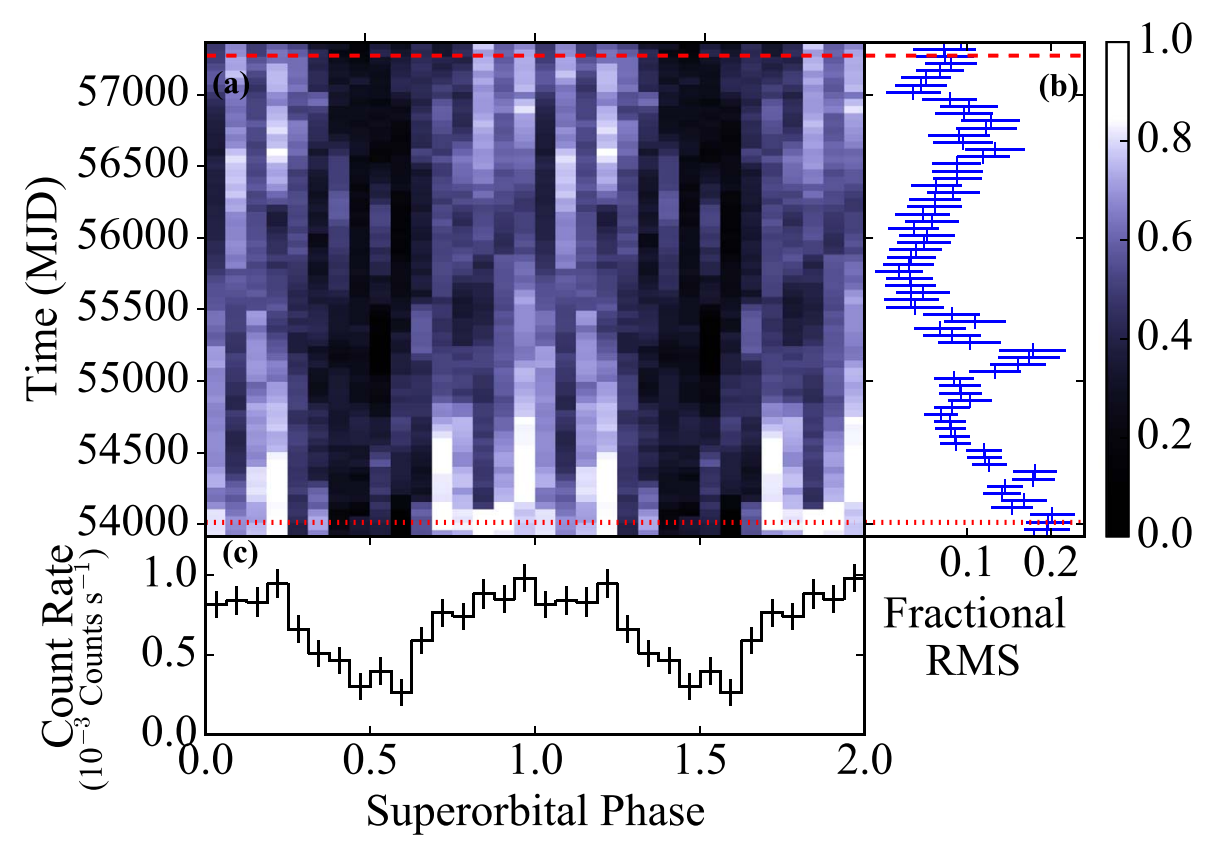

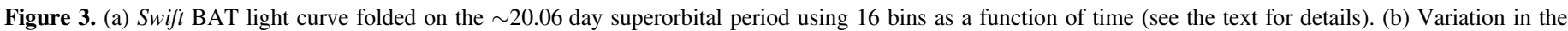

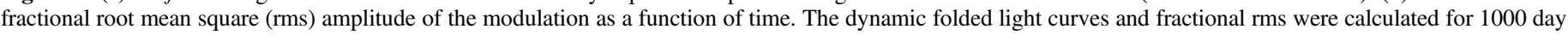

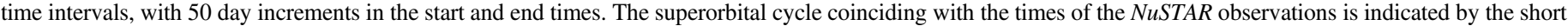

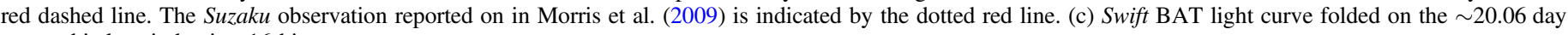
superorbital period using 16 bins.

\subsection{Phase-averaged Spectral Analysis}

The nearly simultaneous NuSTAR and Swift XRT spectra of IGR J16493-4348 were analyzed using the package XSPEC V12.9.1. We made use of the XSPEC convolution model cflux to calculate the fluxes and associated errors. The FPMA and FPMB spectra were simultaneously fit in XSPEC. To account for instrumental calibration uncertainties, we used cross-calibration constants normalized to FPMA during the spectral analysis (see Table 2).

To monitor the spectral evolution of the broadband X-ray emission as a function of superorbital phase, we extracted nearly simultaneous NuSTAR and Swift XRT spectra at superorbital minimum and maximum, respectively (see Figures 8(a) and (e)). For both data sets, we used several models that typically describe systems that host a neutron star: a power law (power), a power law with a high-energy cutoff (highecut; White et al. 1983), a cutoff power-law (cutoffpl), a power law with a Fermi-Dirac cutoff ( $f d c u t$; Tanaka 1986), and a negative-positive exponential cutoff (npex; Makishima et al. 1999). All models were modified by an absorber that fully covers the source (tbabs in XSPEC) using the Verner et al. (1996) cross sections and Wilms et al. (2000) abundances. We note that D'Aì et al. (2011) applied the npex, cutoffpl and fdcut models to a broadband analysis using Swift BAT and INTEGRAL ISGRI data, together with pointed Swift XRT and Suzaku observations, and found the spectra to be best described by the npex model. For our NuSTAR and Swift observations, we could not constrain the cutoff energy with the fdcut model, and found an upper limit at the $90 \%$ confidence interval of $<3 \mathrm{keV}$ at both superorbital minimum and maximum.

We compared the highecut, npex, and cutoffpl residuals at superorbital minimum and maximum in Figures 8(b)-(d) and (f)-(h), respectively, and found that all three models provided a similar quality of fit at superorbital minimum (see Figure 8(a)). The highecut and npex models provided a similar quality of fit at superorbital maximum, but the cutoffpl model yielded a worse $\chi^{2}$ value and wavy residuals (see Figure 8(h)). The results are given in Table 2. We note that for a power law modified by a high-energy cutoff, sharp features can appear as line-like residuals at the cutoff energy. No evidence of such line-like residuals near the cutoff energy was found in either observation (see Figure 8). Unless otherwise noted, we chose to model the spectra using the highecut model since it provided a marginally better fit quality at both superorbital minimum and maximum (see Figures 8(b) and (f)).

The neutral hydrogen column density for the fully covered absorption at superorbital minimum and maximum were found to be $(9 \pm 2) \times 10^{22} \mathrm{~cm}^{-2}$ and $(11 \pm 1) \times 10^{22} \mathrm{~cm}^{-2}$, respectively. These measurements exceed the values reported by the Leiden/Argentine/Bonn survey (Kalberla et al. 2005) and the review by Dickey \& Lockman (1990), which are $1.42 \times$ $10^{22} \mathrm{~cm}^{-2}$ and $1.82 \times 10^{22} \mathrm{~cm}^{-2}$, respectively. This is consistent with absorbing material intrinsic to the source, which is expected for the subclass of obscured SGXBs (Chaty 2011).

Apart from the overall flux change, we find no significant changes in the continuum parameters between superorbital minimum and maximum (see Table 2). Not surprisingly, the unabsorbed X-ray flux is found to increase by more than a factor of 2, where it is found to be $(2.9 \pm 0.3) \times 10^{-11} \mathrm{erg} \mathrm{cm}^{-2} \mathrm{~s}^{-1}$ in the $1-10 \mathrm{keV}$ band near superorbital minimum and $(7.6 \pm 0.2) \times$ $10^{-11} \mathrm{erg} \mathrm{cm}^{-2} \mathrm{~s}^{-1}$ in the $1-10 \mathrm{keV}$ band near superorbital maximum. Assuming a distance of $16.1 \pm 1.5 \mathrm{kpc}$ (Pearlman et al. 2019), the $1-10 \mathrm{keV} \mathrm{X}$-ray luminosity is found to be $(9 \pm 2) \times 10^{35} \mathrm{erg} \mathrm{s}^{-1}$ and $(2.4 \pm 0.5) \times 10^{36} \mathrm{erg} \mathrm{s}^{-1}$ at superorbital minimum and maximum, respectively.

To study the broadband behavior of IGR J16493-4348, we additionally calculated the unabsorbed X-ray flux and luminosity in the $3-40 \mathrm{keV}$ band. At superorbital minimum, the 

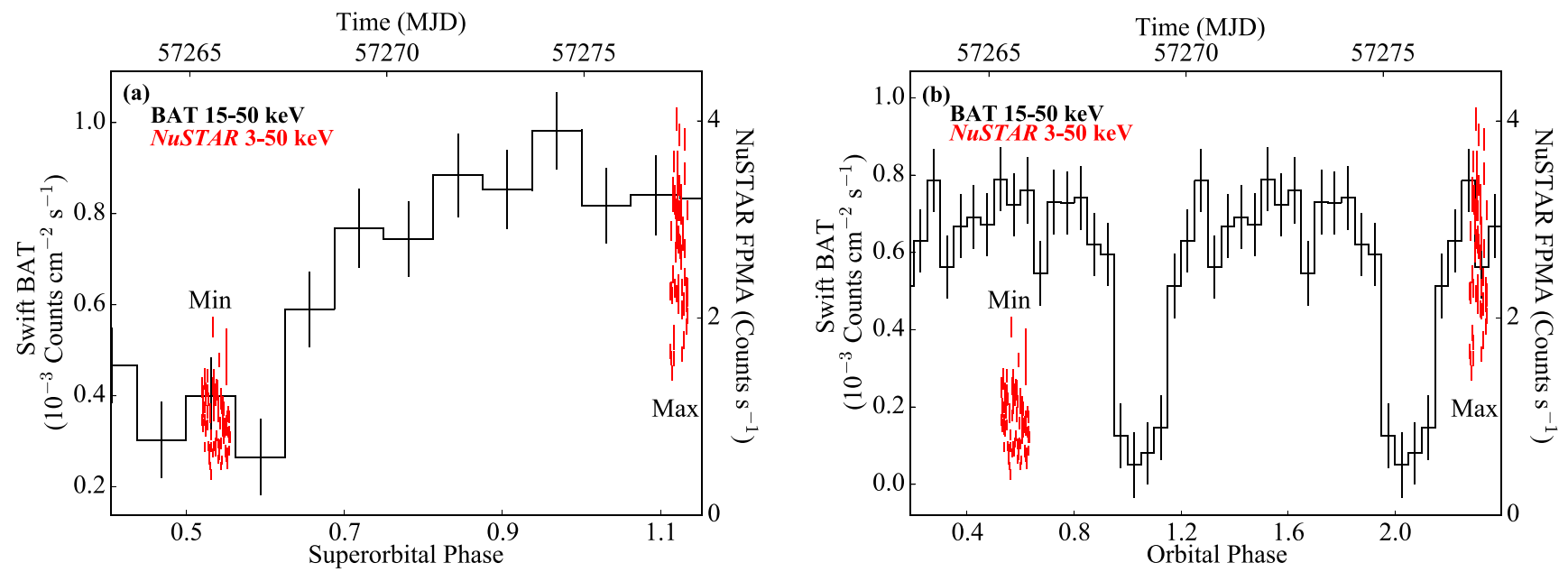

Figure 4. (a) Swift BAT light curve (black) folded on the $\sim 20.06$ day superorbital period using 16 bins. (b) Swift BAT light curve (black) folded on the $\sim 6.7828$ day orbital period using 20 bins. Phase zero for the superorbital and orbital light curves corresponds to the times of maximum flux (see Section 3.1) and maximum delay from the pulsar timing analysis in Pearlman et al. (2019), respectively. The NuSTAR FPMA data near superorbital minimum and maximum are overplotted in red.

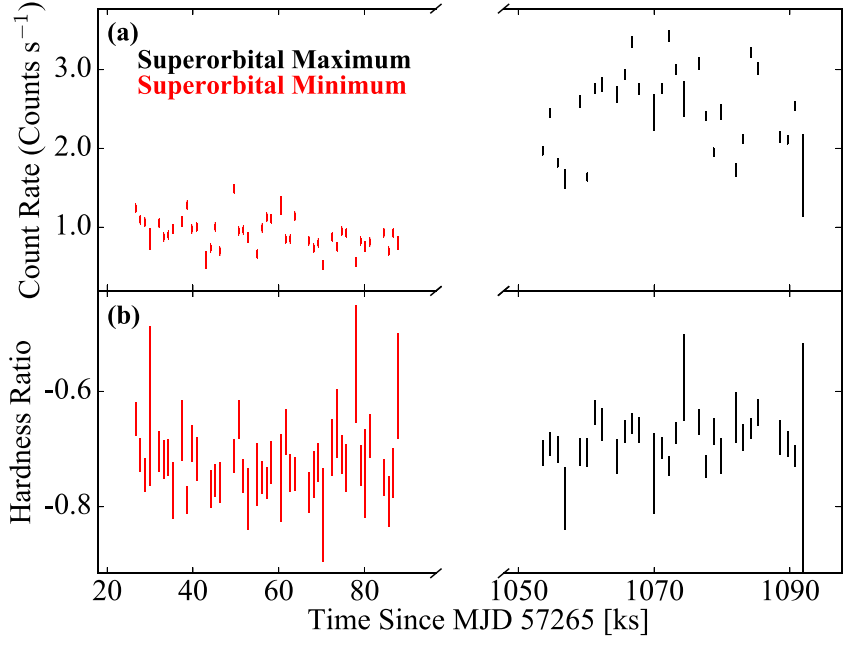

Figure 5. (a) NuSTAR FPMA $3-50 \mathrm{keV}$ light curves and (b) hardness ratio (see the text for details) at superorbital minimum (red) and maximum (black) binned to a time resolution corresponding to the average neutron star rotation period $(\sim 1092.7 \mathrm{~s})$

3-40 keV X-ray flux and luminosity were found to be (4.9 \pm $0.2) \times 10^{-11} \mathrm{erg} \mathrm{cm}^{-2} \mathrm{~s}^{-1}$ and $(1.5 \pm 0.3) \times 10^{36} \mathrm{erg} \mathrm{s}^{-1}$, respectively. The corresponding $\mathrm{X}$-ray flux and luminosity at superorbital maximum were $(1.41 \pm 0.03) \times 10^{-10} \mathrm{erg} \mathrm{cm}^{-2} \mathrm{~s}^{-1}$ and $(4.4 \pm 0.8) \times 10^{36} \mathrm{erg} \mathrm{s}^{-1}$, respectively.

Some residuals were found near $6.4 \mathrm{keV}$ at superorbital maximum, which could indicate a weak $\mathrm{Fe} \mathrm{K} \alpha$ emission feature (see Figure 9). We account for this with a narrow additive Gaussian with a line width fixed to $0.1 \mathrm{keV}$, as the line width was unconstrained by the fit. We also tried freezing the width of the $\mathrm{Fe} \mathrm{K} \alpha$ line to $0.01 \mathrm{keV}$ and $10^{-3} \mathrm{keV}$, but note that it did not significantly affect the best-fit continuum parameters and their uncertainties. This is not surprising since these line widths are smaller than NuSTAR's FWHM energy resolution, which is $400 \mathrm{eV}$ at $6.0 \mathrm{keV}$ (Harrison et al. 2013).

The addition of an $\mathrm{Fe} \mathrm{K} \alpha$ line reduces the $\chi^{2}$ /dof from 807.27/ 755 to $791.14 / 753$. To estimate the significance of the inclusion of an Fe $\mathrm{K} \alpha$ feature to the NuSTAR and Swift spectra, we simulated $10^{4}$ spectra using a Monte Carlo analysis (Protassov et al. 2002). The simulated spectra were modeled without the $\mathrm{Fe} \mathrm{K} \alpha$ emission

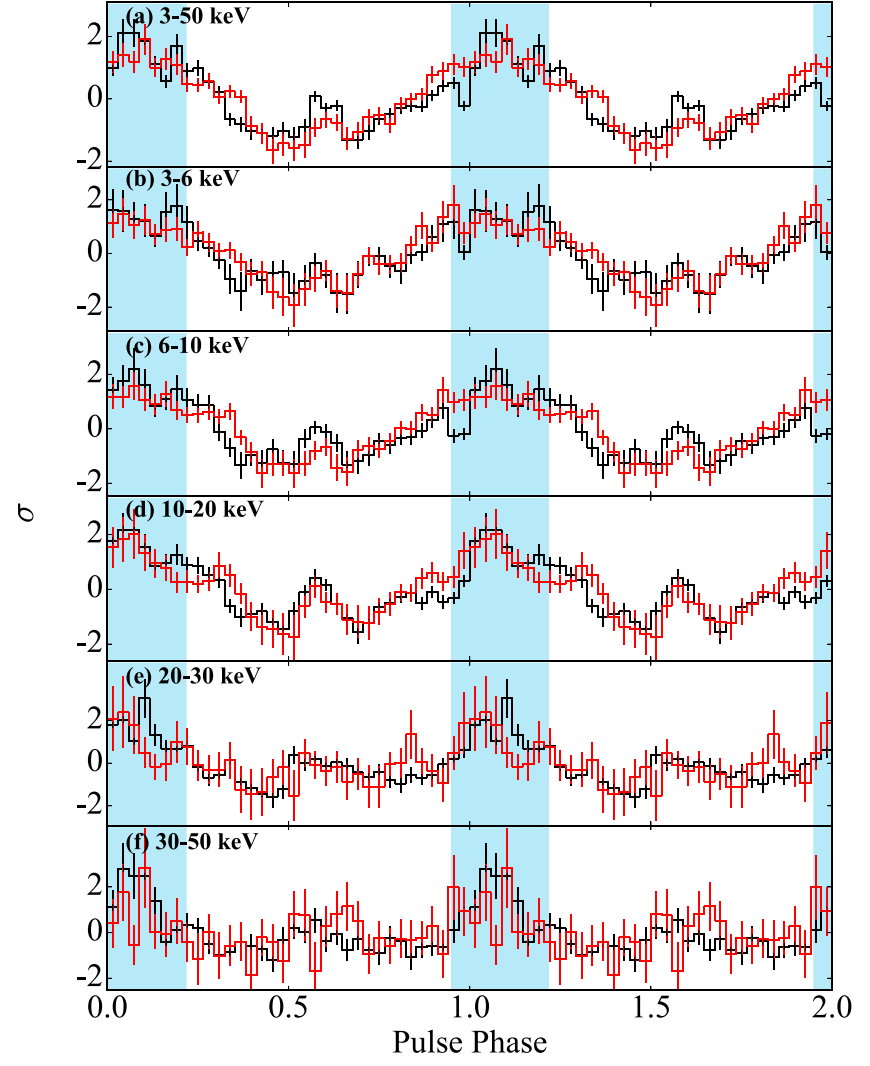

Figure 6. Energy-resolved NUSTAR pulse profiles at superorbital minimum (red) and superorbital maximum (black). The pulse profiles are normalized such that their mean value is zero and their standard deviation is unity. The modulation appears to be double-peaked, with a main broad peak and secondary peak offset by $\sim 0.5$ in phase. The phase range used for the pulsepeak spectral analysis is indicated by the light blue shaded region (see Section 3.5).

component and were fit both with and without the additional component. We compared the difference in simulated $\chi^{2}$ with the observed one, which was found to be 16.1. We found the significance of an $\mathrm{Fe} \mathrm{K} \alpha$ feature to be $99.99 \%$, which supports the presence of a neutral $\mathrm{Fe} \mathrm{K} \alpha$ feature. We found its centroid energy, equivalent width, and flux are $6.36_{-0.10}^{+0.09} \mathrm{keV}, 51_{-19}^{+22} \mathrm{eV}$, and $\left(3_{-2}^{+1}\right) \times 10^{-13} \mathrm{erg} \mathrm{cm}^{-2} \mathrm{~s}^{-1}$, respectively (see Table 2). 


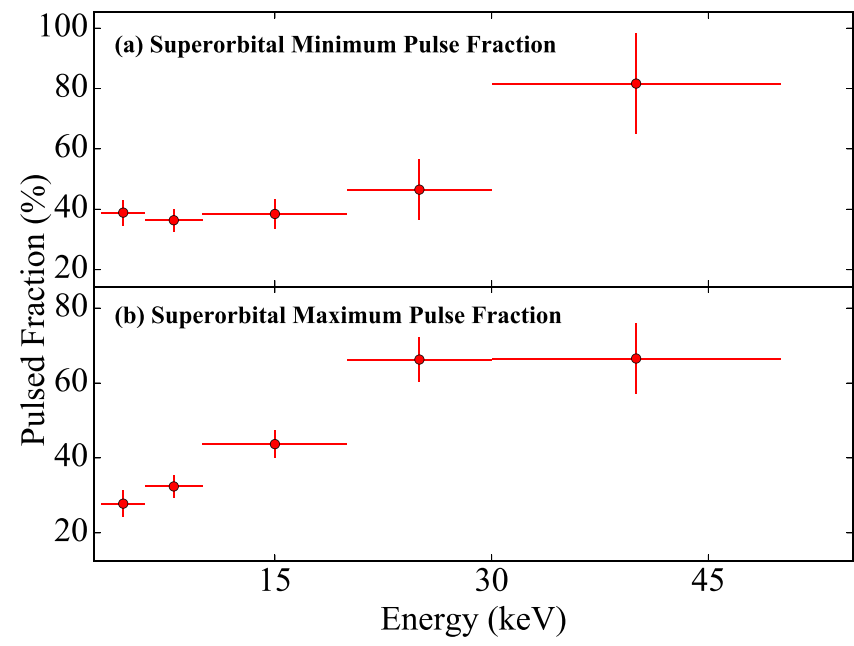

Figure 7. Energy dependence of the pulse fraction of IGR J16493-4348 observed near (a) superorbital minimum and (b) superorbital maximum.

At superorbital minimum, we found the addition of an $\mathrm{Fe} \mathrm{K} \alpha$ feature does not significantly improve the fit quality $\left(\chi^{2} /\right.$ dof changes from 596.17/581 to 596.12/580). Using a similar Monte Carlo analysis, we determined the significance of an $\mathrm{Fe}$ $\mathrm{K} \alpha$ feature to be less than $68 \%$ with $10^{4}$ trials. From a spectral analysis using Suzaku and Swift BAT observations, Morris et al. (2009) found no evidence of strong Fe $\mathrm{K} \alpha$ features and calculated the upper limit of the equivalent width of a $6.4 \mathrm{keV}$ line to be $84 \mathrm{eV}$. To fit the spectrum with the same model in both observations, we chose to include the $\mathrm{Fe} \mathrm{K} \alpha$ line where the energy and width were fixed to the best-fit values found at superorbital maximum. We found an upper limit of $44 \mathrm{eV}$ and $8.0 \times 10^{-14} \mathrm{erg} \mathrm{cm}^{-2} \mathrm{~s}^{-1}$ at the $90 \%$ confidence interval for the equivalent width and flux of the $\mathrm{Fe} \mathrm{K} \alpha$ line, respectively (see Table 2).

Negative residuals were found near $\sim 20 \mathrm{keV}$ at superorbital minimum, indicating the possible presence of a narrow absorption feature (see Figure 8(b)). We modeled the residuals near $\sim 20 \mathrm{keV}$ using a multiplicative line model with the centroid energy, line width, and optical depth as free parameters. We investigated the significance of a $\sim 20 \mathrm{keV}$ feature using the Monte Carlo analysis described in Protassov et al. (2002) and determined its significance to be less than $\sim 60 \%$ with $10^{4}$ trials, which indicates the improvement from adding an absorption component near $\sim 20 \mathrm{keV}$ is negligible. We did not find broad residuals between $30 \mathrm{keV}$ and $40 \mathrm{keV}$ in the NUSTAR spectra, even though such residuals were significant in the BAT and ISGRI spectra reported in D'Aì et al. (2011).

\subsection{Pulse-phase-resolved Spectral Analysis}

We investigated variations in the spectral continuum at different rotational phases of the neutron star using pulsephase-resolved spectroscopy. Since the exposure times of the Swift snapshot observations were short in comparison to the NuSTAR observations (see Table 1), we only considered the NUSTAR spectra for the analysis of phase-dependent changes in the spectral parameters. For the phase-resolved analysis, we chose to subdivide the folded light curves at superorbital minimum and maximum into four equally spaced intervals. We rebinned the phase-resolved spectra using the same procedure as for the phase-averaged spectra (see Section 2.1).
We initially allowed the continuum spectral components described in Table 2 to be free parameters and performed spectral fits on each of the four equally spaced intervals. Since the phase-resolved spectra lack the soft energy coverage made available by Swift XRT, we chose to fix $N_{\mathrm{H}}$ to the phase-averaged values of $9 \times 10^{22} \mathrm{~cm}^{-2}$ and $11 \times 10^{22} \mathrm{~cm}^{-2}$ from the superorbital minimum and maximum spectra, respectively (see Table 2). We also tried to fit the data leaving the fully covered $N_{\mathrm{H}}$ free, but this resulted in large uncertainties in the model parameters.

Figure 10 shows the spectral parameters of the highecut model at different rotational phases of the neutron star. We find possible evidence of an increase in the folding energy between superorbital minimum and maximum near the main peak of the pulse profile (see Figure 10(d)).

In Figure 11, we show the $3 \sigma$ confidence contours between the folding energy and the photon index at both superorbital minimum and maximum at each neutron star rotation period phase bin. The folding energy and photon index are found to be roughly correlated with each other. We found the folding energy significantly increases between superorbital minimum and maximum near the main peak of the pulse profile (see Figure 11(a)-(b)), confirming our results in Figure 10(d).

To investigate possible changes in the pulse-phase-resolved spectral shape between superorbital minimum and maximum, we calculated the hardness ratio using Equation (2). We defined the soft and hard bands to be $3-10 \mathrm{keV}$ and $15-40 \mathrm{keV}$, respectively. The hardness ratio at the main peak of the pulse profile increases with increasing X-ray luminosity (see Figure 10(b)).

Due to the reduced signal-to-noise compared to the phaseaveraged spectrum, the addition of a $6.4 \mathrm{keV}$ emission feature does not significantly improve the quality of the fit in most phase intervals of the phase-resolved spectra at superorbital maximum, even though it was observed at the $99.99 \%$ confidence interval in the superorbital maximum phaseaveraged spectrum (see Section 3.3). We note the $\mathrm{Fe} \mathrm{K} \alpha$ line is significant at the $98.8 \%$ confidence intervals between pulse phases $0.75-1.00$ using a Monte Carlo analysis with $10^{4}$ trials. Since the $\mathrm{Fe} \mathrm{K} \alpha$ line is detected in the phase-averaged spectrum at superorbital maximum, we chose to include it in our pulse-phase-resolved spectra at both superorbital maximum and superorbital minimum with the centroid energy and width frozen to the value determined from the phase-averaged spectrum at superorbital maximum. No fluctuations in the flux of the $\mathrm{Fe} \mathrm{K} \alpha$ line as a function of neutron star rotation period were found (see Figure 10(f)), which could possibly be attributed to the low signal to noise.

\subsection{Pulse-peak Spectral Analysis}

To further investigate possible spectral differences between superorbital minimum and maximum near the broad main peak of the pulse profile, we extracted a spectrum for each observation focusing on pulse phases 0.00-0.22 and 0.88-1.00. We rebinned the pulse-peak-resolved spectra using the same procedure as for the phase-averaged and phaseresolved spectra and again only considered the NuSTAR spectra for the analysis.

For the pulse-peak spectral analysis, we fit the spectra at both superorbital minimum and maximum simultaneously using the procedure described in Kühnel et al. (2016 and references therein). To reduce the number of free parameters, we froze the 
Table 2

Phase-averaged, Broadband X-Ray Spectral Parameters of the Nearly Simultaneous NuSTAR and Swift Observations for Several Empirical Models

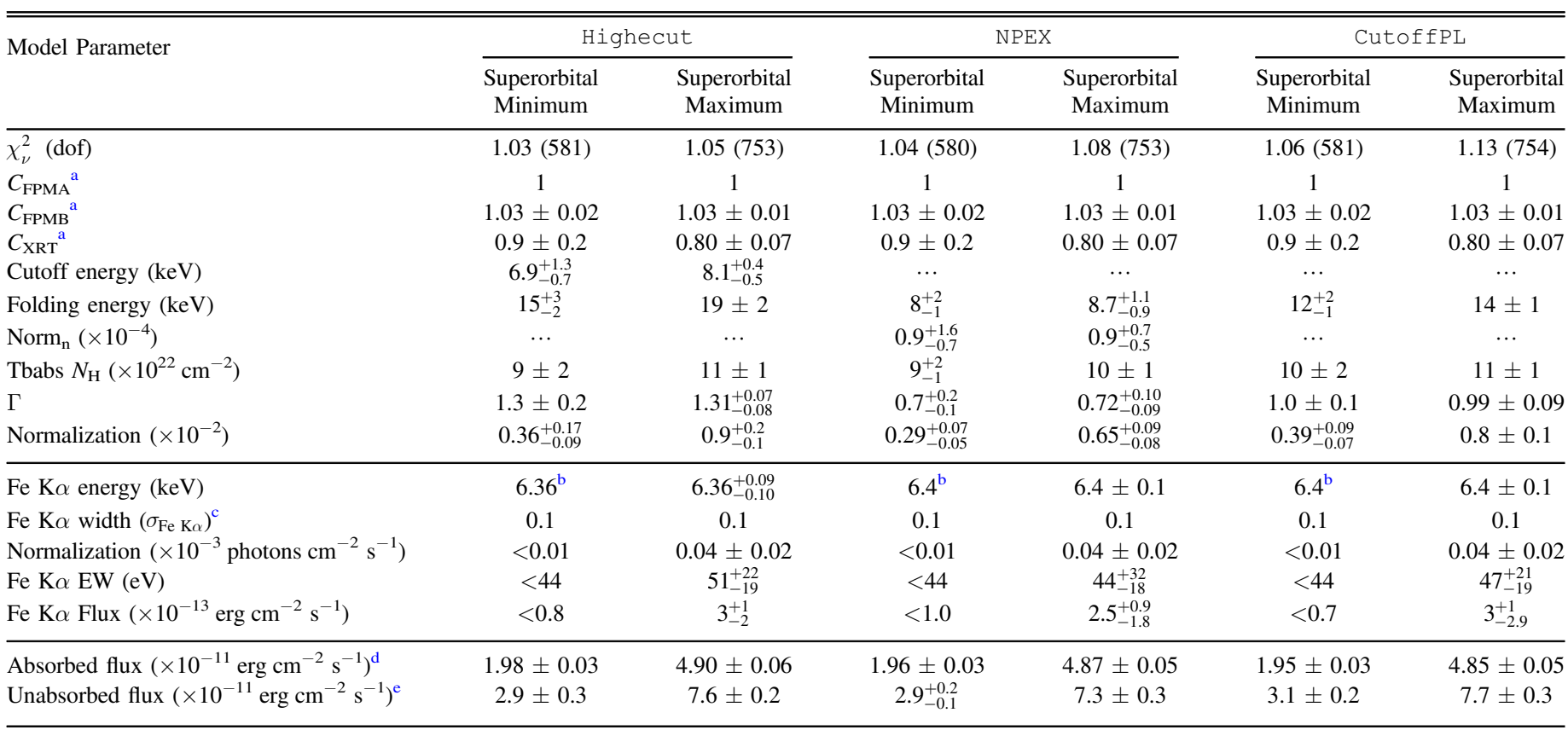

Notes.

${ }^{a}$ Detector cross-calibration constants with respect to FPMA.

$\mathrm{b}$ The energy is frozen because we can only obtain an upper limit.

${ }^{\mathrm{c}}$ The width of the $\mathrm{Fe} \mathrm{K} \alpha$ line is frozen to $0.1 \mathrm{keV}$.

${ }^{\mathrm{d}}$ Absorbed flux in the $1-10 \mathrm{keV}$ band.

${ }^{\mathrm{e}}$ Unabsorbed flux in the $1-10 \mathrm{keV}$ band.

energy and width of the $\mathrm{Fe} \mathrm{K} \alpha$ feature to the phase-averaged value at superorbital maximum. Again, the $\mathrm{Fe} \mathrm{K} \alpha$ feature was not detected at superorbital minimum and we therefore derived an upper limit for the strength of the line.

In Figure 12, we show the pulse-peak NuSTAR FPMA spectra and count rate ratio at superorbital minimum and maximum. At energies above $10 \mathrm{keV}$, we find possible evidence of a harder spectrum at superorbital maximum than at superorbital minimum (see Figure 12(b)). This is consistent with the increase in the hardness ratio from superorbital minimum to superorbital maximum near the peak of the pulse profile (see Figure 10(b)). To investigate changes in the shape of the spectrum between superorbital minimum and maximum, we first fit the pulse-peak spectra at both superorbital phases simultaneously and only allowed the cross-normalization for each spectrum to change. In these fits, the continuum parameters were all tied together to have the same value for the superorbital minimum and maximum spectra (see Brumback et al. 2018 and references therein). We find $\chi_{\nu}^{2}$ to be 1.14 for 838 dof, 1.15 for 838 dof and 1.18 for 837 dof for the highecut, npex, and cutoffpl models, respectively. The cutoff energy, folding energy and photon index for the highecut model were found to be $8.0 \pm 0.3 \mathrm{keV}, 22 \pm 1 \mathrm{keV}$, and $1.35 \pm 0.03$, respectively.

We also fit the pulse-peak spectra at superorbital minimum and maximum, where the folding energy was allowed to vary. This reduces the $\chi_{\nu}^{2}$ for the highecut, npex, and cutoffpl models to 1.01 for 836 dof, 1.02 for 836 dof, and 1.05 for 835 dof, respectively (see Table 3 ). The folding energy for the highecut and cutoffpl models shows a possible increase between superorbital minimum and maximum, which is consistent with our pulse-phase-resolved results (see Figure 10(b)).

We detected possible negative residuals near $\sim 22 \mathrm{keV}$ in the pulse-peak spectrum at superorbital minimum (see Figure 13(a)). To investigate the possibility of an absorption feature, we only fit the pulse-peak spectrum at superorbital minimum and accounted for the residuals with a multiplicative Gaussian absorption feature. The addition of an absorption line reduces the $\chi^{2} /$ dof for the highecut model from $324.69 / 314$ to $314.82 / 312$ (see Figures 13(b) and (c)). We note the width of the feature cannot be constrained and instead we find the upper limit of the width to be $0.4 \mathrm{keV}$. The energy and optical depth of the possible absorption feature for the highecut model are found to be $23.1 \pm 0.4 \mathrm{keV}$ and $0.8_{-0.2}^{+0.4}$, respectively. We do not find a significant change in the spectral parameters if instead we use a Lorentzian optical depth profile to describe the possible absorption line. In this case, the $\chi^{2} /$ dof for the highecut model is reduced from $324.69 / 314$ to $315.07 / 312$.

We also investigated the possibility that the choice of the continuum influences the energy and shape of the possible absorption feature. For the npex and cutoffpl models, the addition of an absorption line reduces the $\chi^{2} /$ dof from $330.15 /$ 314 to $319.91 / 312$ and $338.40 / 315$ to $329.17 / 313$, respectively (see Figures $13(\mathrm{~d})-(\mathrm{g})$ ). The energy of the line is found to be $23.3 \pm 0.4 \mathrm{keV}$ and $23.3 \pm 0.5 \mathrm{keV}$ for the npex and cutoffpl models, respectively.

To determine the significance of the inclusion of the possible absorption feature, we simulated $10^{4}$ spectra using the Monte Carlo analysis described in Protassov et al. (2002). Unlike our 

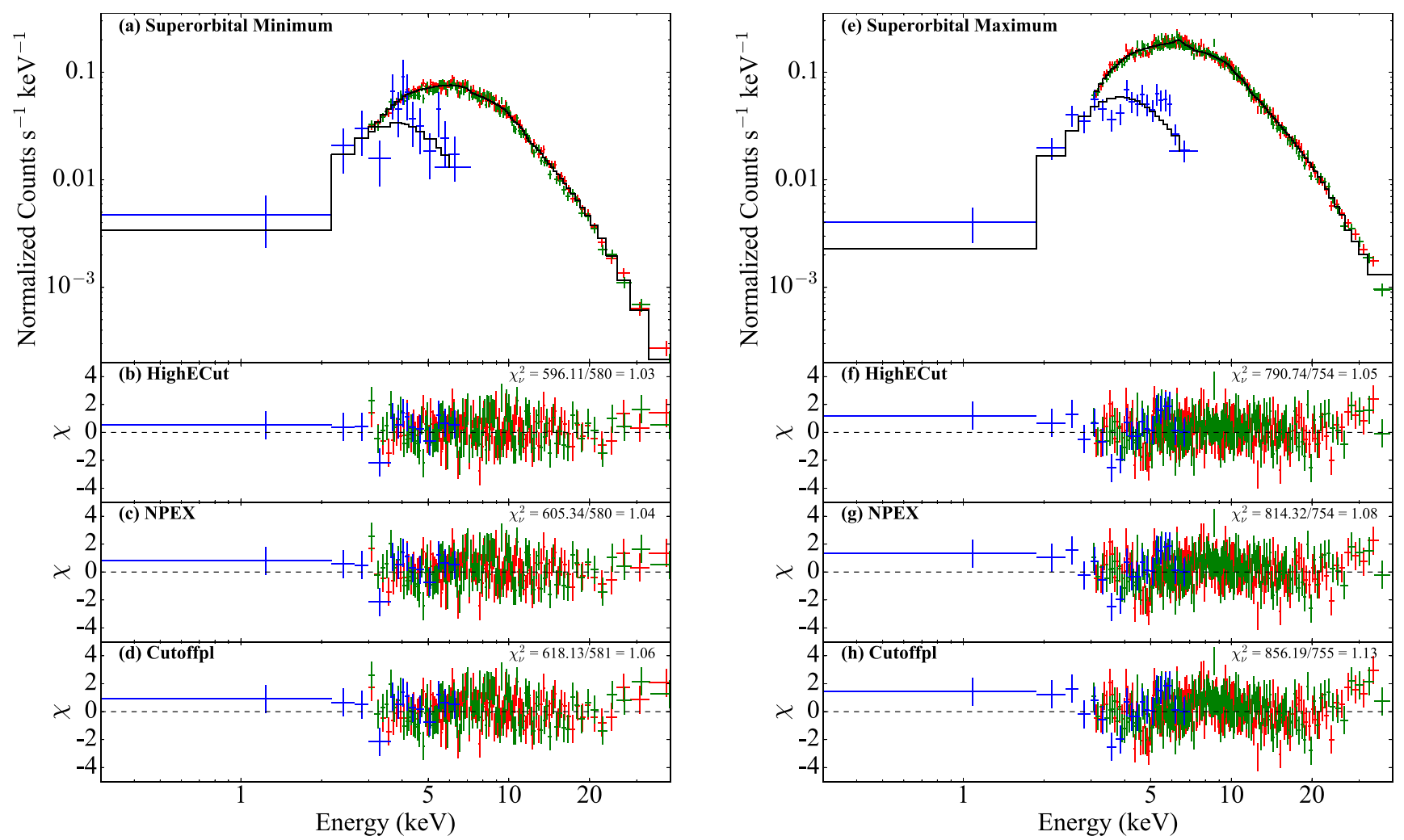

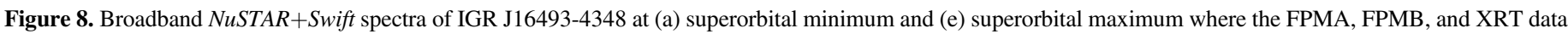

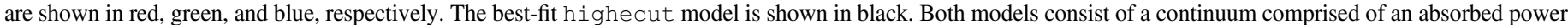

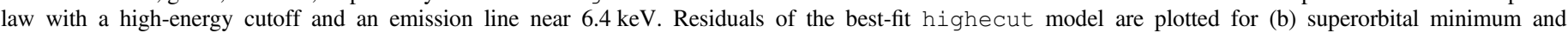

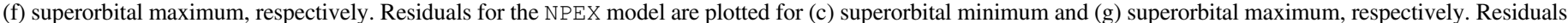
for the cutoffpl model are plotted for (d) superorbital minimum and (h) superorbital maximum, respectively. The spectra are rebinned for clarity.

Monte Carlo simulations for $\mathrm{Fe} \mathrm{K} \alpha$ where we restricted the energy to that of the best-fit model (see Sections 3.3 and 3.4), we allowed the energy of the possible absorption feature to vary between $10 \mathrm{keV}$ and $40 \mathrm{keV}$. These are reasonable values for a possible CRSF (Coburn et al. 2002). We determined the probability of the possible absorption feature arising by chance to be $36.4 \%$ with $10^{4}$ trials, which shows that the feature is not significant.

\section{Discussion}

\subsection{Spectral Evolution as a Function of Superorbital Period}

In Figure 14, we plot the photon index and folding energy of the pulse-phase-averaged NuSTAR and Swift XRT data as a function of the $1-10 \mathrm{keV}$ X-ray luminosity. For the highecut model, the pulse-phase-averaged X-ray luminosity of IGR J16493-4348 increased from $(9 \pm 2) \times 10^{35} \mathrm{erg} \mathrm{s}^{-1}$ at superorbital minimum to $(2.4 \pm 0.4) \times 10^{36} \mathrm{erg} \mathrm{s}^{-1}$ at superorbital maximum (see Section 3.3). To place the data in context, we also show the change in the photon index and folding energy with respect to $1-10 \mathrm{keV}$ X-ray luminosity of the broadband Swift BAT and the INTEGRAL ISGRI, together with pointed Swift XRT and Suzaku observations reported in D'Aì et al. (2011). The Swift XRT and Suzaku observations of IGR J16493-4348 took place at MJD 53,805.9-53,806.4 and MJD 54,013.9-54,014.4, respectively; which correspond to superorbital phases $\sim 0.05-0.07$ and $\sim 0.42-0.45$ or slightly earlier superorbital phases than our NUSTAR campaign. Their $\mathrm{X}$-ray luminosities, however, are similar to our NuSTAR observations (see Figure 14).
In accreting X-ray pulsars, the shape of the pulse profiles has been found to depend on the emission processes and the relative contribution of the two accretion columns (Meszaros \& Nagel 1985; Kraus et al. 1989; Falkner et al. 2019). Our NuSTAR observations of IGR J16493-4348 show that, despite the increase in X-ray flux, the pulse profiles show no significant changes in shape between superorbital minimum and maximum. The pulse profiles in both observations were found to weakly depend on energy and be double-peaked in structure (see Figure 6). This may indicate that the emission properties in the accretion column may not change between the two superorbital phases (e.g., A 0535+26; Ballhausen et al. 2017). Using RXTE PCA data that span times between MJD 55,843.1 and MJD 55,852.6, corresponding to superorbital phases 0.62-0.09, Pearlman et al. (2019) also found a double-peaked shape of the pulse profiles and a weak energy dependence. They also observed the pulsed fraction to increase with increasing energy, which we confirm with $N U S T A R$ (see Figure 7). We suggest the similar properties of pulse profiles as seen by both NUSTAR and RXTE may be linked to an accretion regime that does not change between superorbital minimum and maximum (see Postnov et al. 2015 and references therein).

In X-ray binaries that host accretion powered pulsars, the physical conditions inside the accretion column depend on the mass accretion rate. The resulting X-ray emission can be characterized in terms of the local Eddington limit $\left(L_{\text {crit }}\right.$, Becker et al. 2012), which for a magnetic dipole geometry is proportional to the magnetic field strength. As indicated in Section 1, the magnetic field of the neutron star is often directly measured by CRSFs. We do not find any significant CRSFs in 


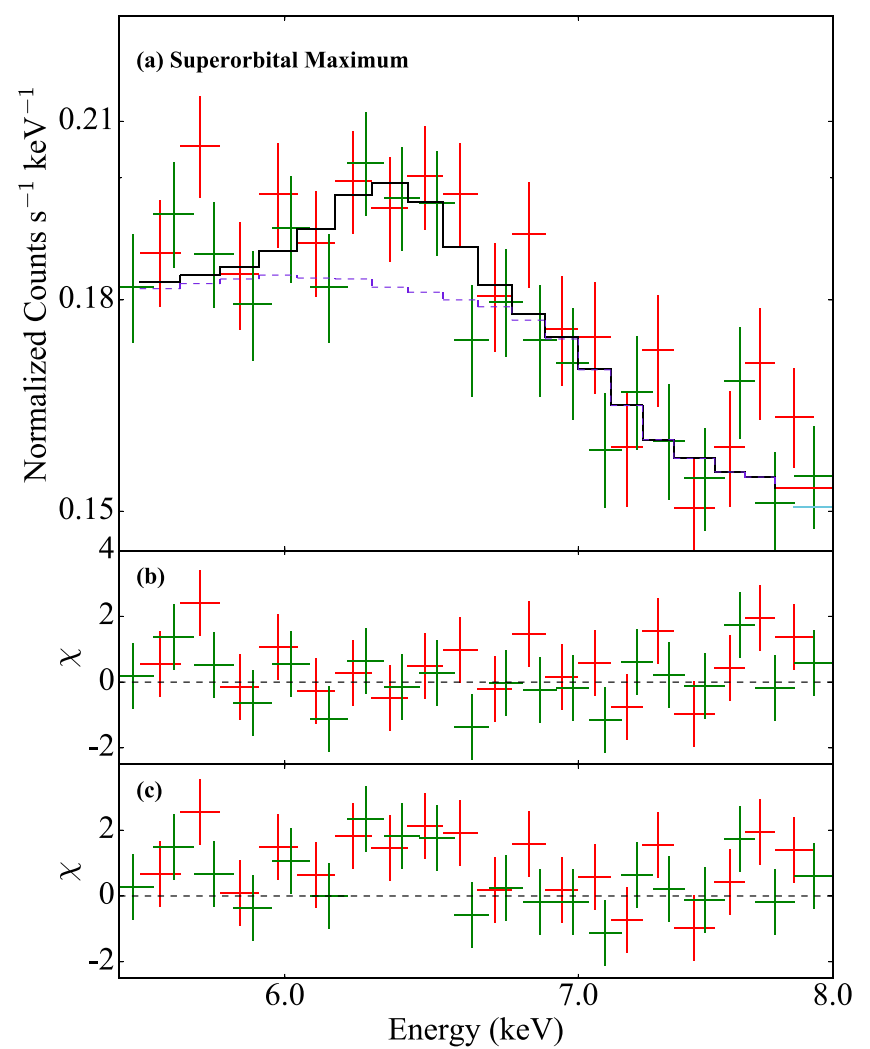

Figure 9. FPMA+FPMB spectrum of IGR J16493-4348 between 5.5-8.0 keV band at superorbital maximum. The Fe $\mathrm{K} \alpha$ emission line is shown along with the best-fit model (black). (a) FPMA and FPMB data indicated by the red and green data points. The dashed purple line indicates the fit without the $\mathrm{Fe} \mathrm{K} \alpha$ emission line. (b) Residuals with the $\mathrm{Fe} \mathrm{K} \alpha$ emission line included in the spectral model. (c) Residuals without the Fe $\mathrm{K} \alpha$ emission line included in the spectral model.

the pulse phase-averaged or phase-resolved NuSTAR spectra of IGR J16493-4348, which is a possible indication that the magnetic field might be predominately seen under small viewing angles (Schwarm et al. 2017).

At low to intermediate accretion rates, the $\mathrm{X}$-ray luminosity is below the critical value where radiation pressure becomes important. The in-falling matter may be primarily decelerated by Coulomb interactions, and is thought to form an accretion mound close to the surface of the neutron star (Postnov et al. 2015 and references therein). Our NuSTAR results show that the X-ray luminosity observed in IGR J16493-4348 is on the order of $10^{35}-10^{36} \mathrm{erg} \mathrm{s}^{-1}$, which is consistent with this picture (see Becker et al. 2012 and references therein).

The spectral shape in X-ray binary pulsars that are accreting at low to intermediate accretion rates is observed to harden toward higher X-ray fluxes (e.g., Her X-1, A 0535+26; Klochkov et al. 2011; Ballhausen et al. 2017). Our pulse-phaseresolved NUSTAR results of IGR J16493-4348 near the broad main peak of the pulse profile show that the pulse-phaseresolved hardness ratio increases between superorbital minimum and maximum (see Figure 10(b)), which is a possible indication that the spectrum hardens with increasing X-ray flux. This is also supported by the ratio of the two peak spectra, which shows a clear slope, indicating the increasing dominance of the flux at superorbital maximum toward higher energies (see Figure 12(b)).

It is interesting to note that D'Aì et al. (2011) found the photon index flattens between superorbital minimum and

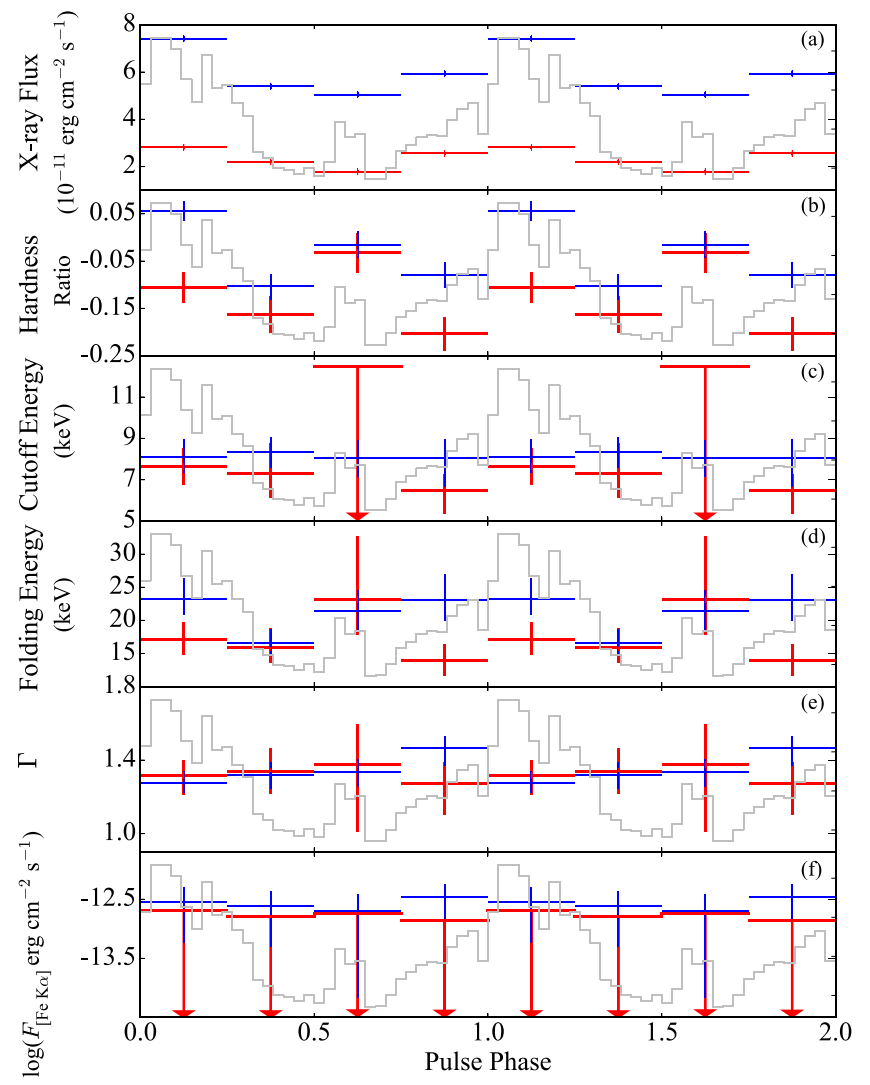

Figure 10. NUSTAR spectral parameters as a function of pulse phase using the power law with high-energy cutoff model. The observations at superorbital minimum and maximum are indicated by the red and blue points, respectively. The gray histogram shows the $N u S T A R$ light curve at superorbital maximum in the $3-50 \mathrm{keV}$ energy range folded on the neutron star rotation period.

maximum (see Figure 14(a)). While this may also suggest that the spectral shape of IGR J16493-4348 hardens with increasing $\mathrm{X}$-ray flux, the photon indices observed with NuSTAR were found to be consistent between the superorbital minimum and maximum. As shown in Figure 14, they were additionally found to be consistent with those reported in D'Aì et al. (2011). It should also be noted that D'Aì et al. found the folding energy in their broadband BAT and ISGRI, together with pointed Swift XRT and Suzaku observations, to be systematically higher than those we derived with NuSTAR (see Figure 14(b)). These differences may result from the fact that the spectral shape may have changed between the observations reported in D'Aì et al. and our NUSTAR observations. Another possible reason is the energy gap between the soft (Swift XRT and Suzaku) and hard (INTEGRAL and Swift BAT) bands, which may affect the spectral fits reported in D'Aì et al.

This observed spectral hardening seen near the main peak of the pulse profile could possibly be explained by Comptonsaturated emission from the sidewall of the optically thick accretion column (Postnov et al. 2015). We found the folding energy near the main pulse peak to increase between superorbital minimum and maximum, but no change in the photon index was found (see Table 3). This may suggest the average temperature of the Comptonizing gas increases between superorbital minimum and maximum. Due to this temperature increase, the plasma more efficiently upscatters photons to higher energies via the inverse Compton effect, resulting in a harder observed spectrum as is observed in the pulse-peak superorbital maximum spectrum. 


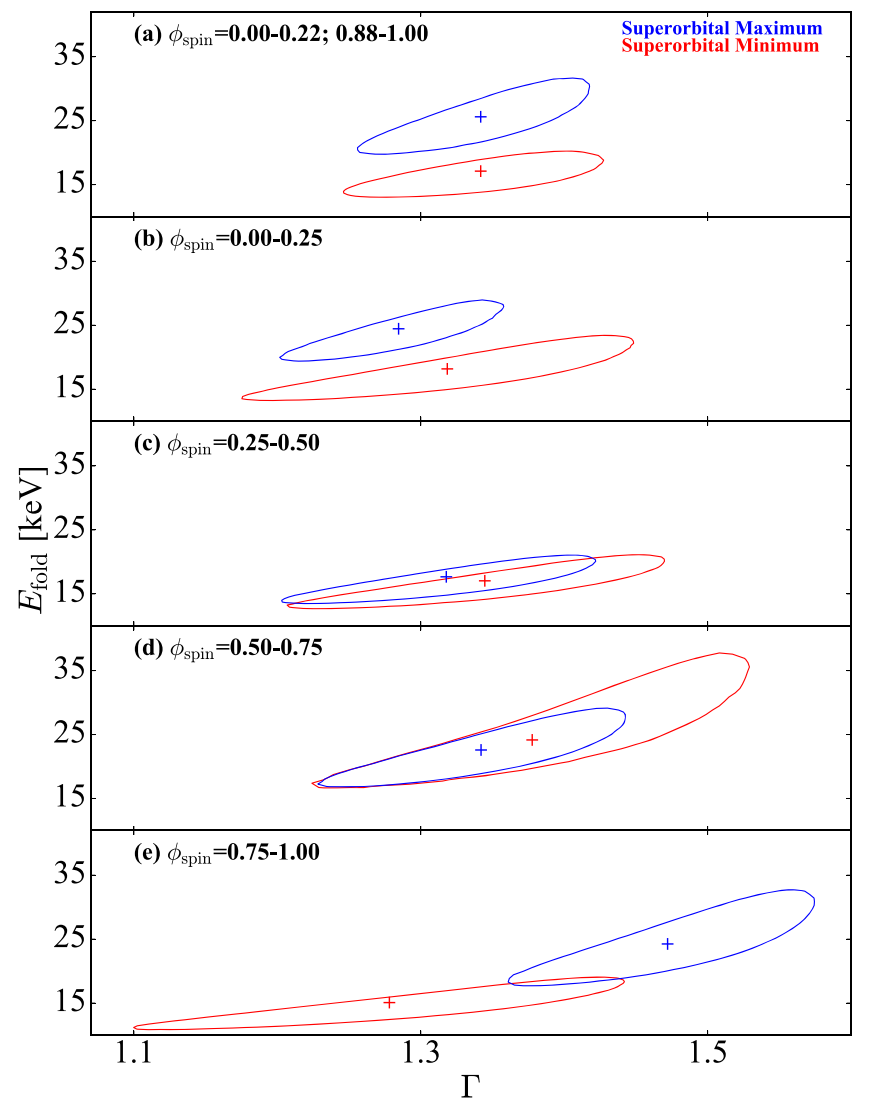

Figure 11. Contours of the folding energy, $E_{\mathrm{fold}}$, and photon index, $\Gamma$, at the $3 \sigma$ confidence interval for the phase-resolved spectra at superorbital minimum (red) and maximum (blue). The neutral hydrogen column density, Fe $\mathrm{K} \alpha$ energy, and detector calibration constants were held constant. The best-fit values at superorbital minimum and maximum are indicated by the red and blue crosses, respectively.

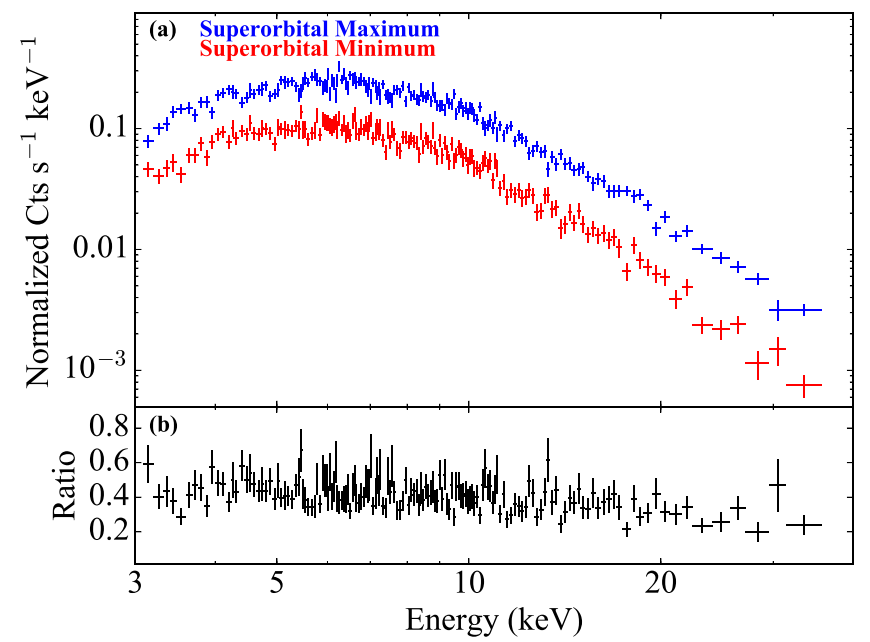

Figure 12. (a) Pulse-peak FPMA spectra at superorbital minimum (red) and superorbital maximum (blue). The superorbital maximum spectrum was rebinned to match the energy binning of the superorbital minimum spectrum for the plot to compare the residuals on a bin-by-bin basis. (b) Count rate spectral ratio between superorbital minimum and maximum (see the text for details).

\subsection{Comparison with $2 S 0114+650$}

To place IGR J16493-4348 in context with other wind-fed SGXBs where superorbital variability is found, we compare it with $2 \mathrm{~S} 0114+650$. This is a wind-fed SGXB where a
30.7 day superorbital period was found (Farrell et al. 2006). The spectral type of the mass donor in $2 \mathrm{~S} 0114+650$ was found to be B1 I (Reig et al. 1996), which is similar to the B0.5 Ia spectral type in IGR J16493-4348 (Pearlman et al. 2019), and its distance was estimated to be $7.2 \mathrm{kpc}$.

We first discuss the Swift BAT observations of IGR J164934348 in comparison to the long-term monitoring of the 30.7 day cycle present in $2 \mathrm{~S} 0114+650$. Using RXTE All-Sky Monitor (ASM) data that spanned $\sim 8.5 \mathrm{yr}$, Farrell et al. (2006) found that the amplitude of the 30.7 day modulation changed as a function of time. This was recently confirmed by $\mathrm{Hu}$ et al. (2017) using ASM and BAT data spanning 20 yr. No significant changes in its frequency were found, which is similar to what we find for IGR J16493-4348.

We also discuss pointed observations of $2 \mathrm{~S} 0114+650$ and how they compare with our joint $N u S T A R$ and Swift campaign for IGR J16493-4348. In their RXTE campaign, which covered two cycles of the $\sim 30.7$ day period, Farrell et al. (2008) found no changes in the intrinsic neutral column density on superorbital timescales. The spectral shape in $2 \mathrm{~S} 0114+650$ was found to harden as the superorbital cycle progressed from minimum to maximum (Farrell et al. 2008), which is similar to our pulse-peak analysis of IGR J16493-4348 (see Sections 3.4-3.5). We note Farrell et al. (2008) have shown that the photon index increases by a factor of two between superorbital maximum and minimum in $2 \mathrm{~S} 0114+650$, which is not seen in our observations of IGR J16493-4348.

Although 2S 0114+650 and IGR J16493-4348 are both mediated by wind accretion, their superorbital modulations may show somewhat different spectral behavior. The spectrum of $2 \mathrm{~S} \quad 0114+650$ significantly hardened toward higher luminosities, but the correlation between spectral hardness and X-ray luminosity in IGR J16493-4348 may be weaker and is observed only near the peak of the pulse profile (see Sections 3.4-3.5). To investigate these possible differences between 2S 0114+650 and IGR J16493-4348, we compare the accretion regimes between the two sources.

Farrell et al. (2008) found that the average absorbed $3-50 \mathrm{keV}$ $\mathrm{X}$-ray flux of $2 \mathrm{~S} 0114+650$ was $2.3 \times 10^{-10} \mathrm{erg} \mathrm{cm}^{-2} \mathrm{~s}^{-1}$ and the fully covered absorption to be $3.2_{-0.8}^{+0.9} \times 10^{22} \mathrm{~cm}^{-2}$. To investigate whether IGR J16493-4348 and 2S 0114+650 are accreting in similar accretion regimes, we calculated the unabsorbed X-ray luminosity in $2 \mathrm{~S} 0114+650$. Assuming the spectral parameters reported in Farrell et al. (2008), we corrected for absorption using PIMMS and found the unabsorbed $3-50 \mathrm{keV}$ $\mathrm{X}$-ray flux in $2 \mathrm{~S} 0114+650$ to be $2.4 \times 10^{-10} \mathrm{erg} \mathrm{cm}^{-2} \mathrm{~s}^{-1}$. The average $3-50 \mathrm{keV} \mathrm{X}$-ray luminosity of $2 \mathrm{~S} 0114+650$ is found to be $1.5 \times 10^{36} \mathrm{erg} \mathrm{s}^{-1}$, which is of the same order of magnitude as the average 3-50 keV X-ray luminosity observed in IGR J164934348 with NuSTAR and Swift XRT (see Section 3.3). Our results near the peak of the pulse profile show that changes in the spectral shape of IGR J16493-4348 are similar to those of 2S 0114+650, albeit the trend in IGR J16493-4348 is somewhat weaker.

\subsection{Superorbital Modulation in ULX Sources}

Superorbital modulation on timescales of tens of days has also been detected in ULX pulsars (e.g., NGC 5907 ULX1, NGC 7793 P13, M82 X-2; Walton et al. 2016; Fürst et al. 2018; Brightman et al. 2019), accreting neutron stars with apparent luminosities in excess of $10^{39} \mathrm{erg} \mathrm{s}^{-1}$. While such timescales are similar to those seen in X-ray binaries accreting at sub-Eddington rates, it is important to note that the timing 
Table 3

Pulse-peak-resolved Broadband X-Ray Spectral Parameters

\begin{tabular}{|c|c|c|c|c|c|c|}
\hline Model Parameter & \multicolumn{2}{|c|}{ Highecut } & \multicolumn{2}{|c|}{ NPEX } & \multicolumn{2}{|c|}{ CutoffPL } \\
\hline$C_{\mathrm{FPMA}}$ & $1^{\mathrm{a}}$ & $2.44 \pm 0.06^{\mathrm{a}}$ & $1^{\mathrm{a}}$ & $2.12 \pm 0.08^{\mathrm{a}}$ & $1^{\mathrm{a}}$ & $2.13 \pm 0.09^{\mathrm{a}}$ \\
\hline$C_{\mathrm{FPMB}}$ & $1.03 \pm 0.02^{\mathrm{a}}$ & $2.51 \pm 0.06^{\mathrm{a}}$ & $1.03 \pm 0.02^{\mathrm{a}}$ & $2.18_{-0.08}^{+0.09 a}$ & $1.03 \pm 0.02^{\mathrm{a}}$ & $2.19 \pm 0.09^{\mathrm{a}}$ \\
\hline Cutoff energy (keV) & \multicolumn{2}{|c|}{$7.7_{-0.9}^{+0.7}$} & $\ldots$ & $\ldots$ & $\ldots$ & $\ldots$ \\
\hline Tbabs $N_{\mathrm{H}}\left(\times 10^{22} \mathrm{~cm}^{-2}\right)^{\mathrm{b}}$ & 9 & 11 & 9 & 10 & 10 & 11 \\
\hline$\Gamma$ & \multicolumn{2}{|c|}{$1.34_{-0.07}^{+0.05}$} & \multicolumn{2}{|c|}{$0.79_{-0.08}^{+0.09}$} & \multicolumn{2}{|c|}{$1.10 \pm 0.06$} \\
\hline Normalization $\left(\times 10^{-2}\right)$ & $0.48_{-0.05}^{+0.04}$ & $1.2 \pm 0.1$ & $0.38 \pm 0.03$ & $0.81_{-0.07}^{+0.08}$ & $0.51 \pm 0.04$ & $1.09_{-0.09}^{+0.10}$ \\
\hline Fe K $\alpha$ energy $(\mathrm{keV})^{\mathrm{b}}$ & \multicolumn{2}{|c|}{6.36} & \multicolumn{2}{|c|}{6.4} & \multicolumn{2}{|c|}{6.4} \\
\hline $\mathrm{Fe} \mathrm{K} \alpha$ width $\left(\sigma_{\mathrm{Fe} \mathrm{K} \alpha}\right)^{\mathrm{b}}$ & \multicolumn{2}{|c|}{0.1} & \multicolumn{2}{|c|}{0.1} & \multicolumn{2}{|c|}{0.1} \\
\hline Unabsorbed flux $\left(\times 10^{-11} \mathrm{erg} \mathrm{cm}^{-2} \mathrm{~s}^{-1}\right)^{\mathrm{d}}$ & $2.87 \pm 0.04$ & $7.0 \pm 0.2$ & $2.90 \pm 0.04$ & $7.0 \pm 0.2$ & $2.94 \pm 0.06$ & $7.1 \pm 0.2$ \\
\hline
\end{tabular}

Notes.

${ }^{\text {a }}$ Cross-normalizations between detectors are calculated with respect to the value of FPMA at superorbital minimum.

b The neutral hydrogen absorption column density, $\mathrm{Fe} \mathrm{K} \alpha$ line energy and width are frozen to the phase-averaged values at superorbital maximum.

c Absorbed flux in the $3-10 \mathrm{keV}$ band.

${ }^{\mathrm{d}}$ Unabsorbed flux in the $3-10 \mathrm{keV}$ band.

and spectral properties of ULXs show significant differences compared to those observed in wind-fed SGXBs such as IGR J16493-4348. Due to their super-Eddington X-ray luminosities, the mode of accretion in ULX pulsars has been ascribed to Roche-lobe overflow (Bachetti et al. 2014) and the superorbital mechanism is likely to be partially driven by a precessing accretion disk (see Fürst et al. 2017 and references therein). The modulation amplitude between superorbital minimum and maximum in ULX pulsars show similarities with X-ray binaries where superorbital variations are driven by a precessing disk. For example, the amplitude of the 60 day modulation observed in M82 X-2 was found to vary by two orders of magnitude (Brightman et al. 2019), which is similar the variability observed in LMC X-4 (Molkov et al. 2015).

Some ULX pulsars have also been observed to exhibit "off" states, where their X-ray fluxes were found to be up to several orders of magnitude lower than expected from an extrapolation of their observed periodic signals (Walton et al. 2016). While the amplitude of the superorbital modulation was found to significantly change on long timescales (see Figure 2), no evidence of "off" states was revealed in our Swift BAT observations in IGR J16493-4348.

\subsection{Superorbital Modulation Mechanism}

\subsubsection{Precessing Accretion Disk}

We first discuss the possibility that the $\sim 20$ day modulation in IGR J16493-4348 is driven by a precessing accretion disk (e.g., Her X-1; Scott et al. 2000). Large variations in the intrinsic neutral hydrogen absorption column are found in this case, (e.g., Her X-1; Ramsay et al. 2002) resulting in sharp dips in the superorbital profile (e.g., Her X-1, SMC X-1; Klochkov et al. 2006; Trowbridge et al. 2007). No significant changes in
$N_{\mathrm{H}}$ are observed between superorbital minimum and maximum (see Table 2), providing evidence against this model. Dramatic changes in the strength of X-ray pulsations have also been found in sources where superorbital modulation is linked to the precession of an accretion disk such as SMC X-1 (Pike et al. 2019) and ULX pulsars (e.g., M82 X-2; Bachetti et al. 2014). In these sources, the variations in the strength of the pulsations were not accompanied by large changes in X-ray flux. No such changes in the pulse profile and pulsed fraction that can be explained by variations in absorption are observed in IGR J16493-4348, which suggests that the 20 day modulation is probably not driven by a precessing accretion disk.

If the $\sim 20.6$ day modulation was driven by a moderately long-lived prograde transient accretion disk, long-term variations in its modulation amplitude may be observable (e.g., $2 \mathrm{~S}$ 0114+650; $\mathrm{Hu}$ et al. 2017). In the BAT dynamic power spectrum, we found a low amplitude in the superorbital modulation spanning $\sim 600$ days (see Figure 2 ), which is similar to the formation and dissipation timescale of a transient accretion disk proposed to be present in $2 \mathrm{~S} 0114+650$. We note in systems where a transient disk may be present, the neutron star is expected to rapidly spin up due to the large angular momentum transfered to it (e.g., OAO 1657-415; Jenke et al. 2012). Pearlman et al. (2019) found no evidence of a rapid spin-up torque in their pulsar timing analysis using the $R X T E$ PCA, which suggests that a transient accretion disk may not be present.

\subsubsection{Stellar Triple System}

Next, we discuss the possible case that IGR J16493-4348 is part of a triple-star system (e.g., 4U 1820-30; Chou \& Grindlay 2001). In a triple-star system, the eccentricity of the 


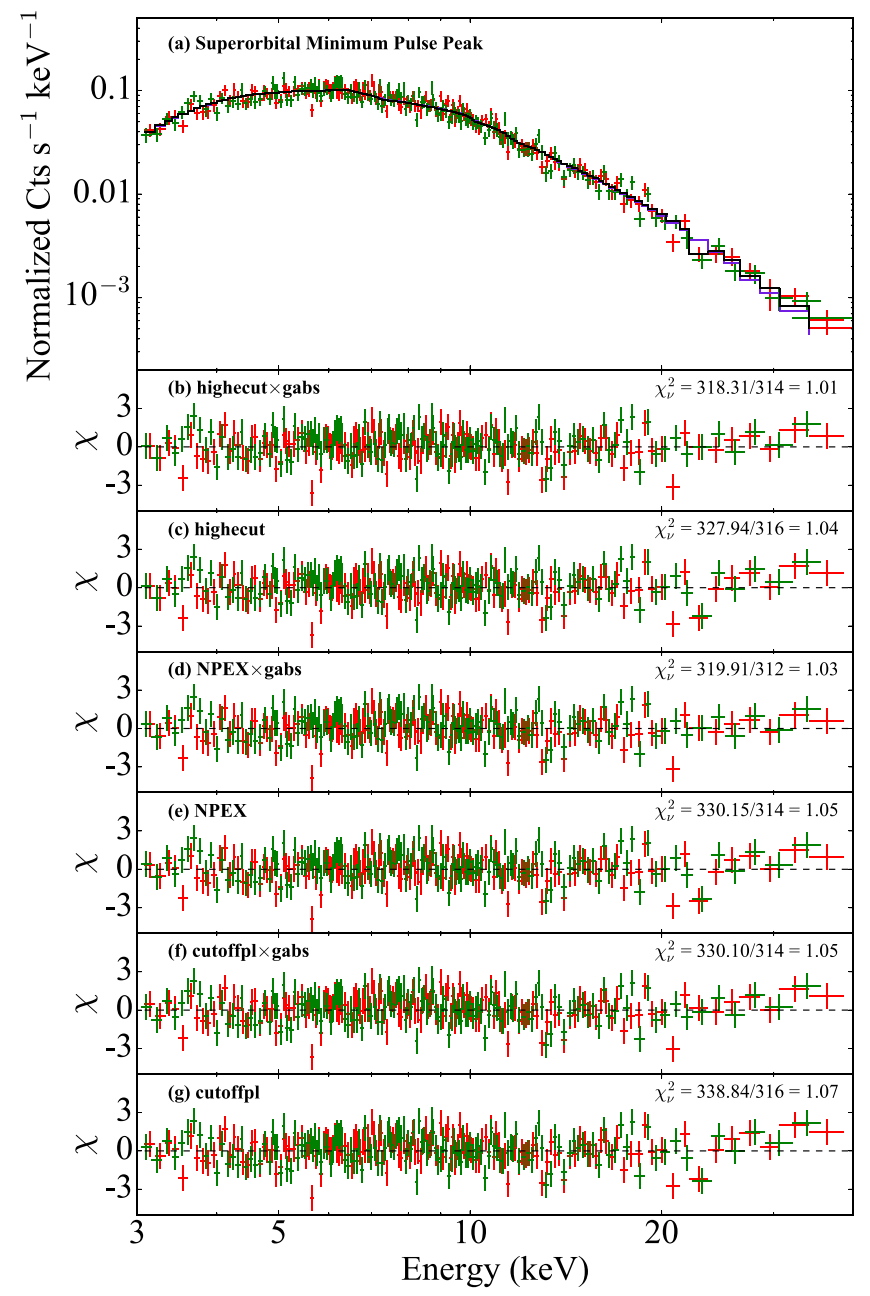

Figure 13. (a) Pulse-peak NUSTAR spectra of IGR J16493-4348 at superorbital minimum. The FPMA and FPMB data are shown in red and green, respectively. The highecut model is shown in black. (b) Residuals for the highecut model with the possible absorption line modeled by a Gaussian profile included in the model. (c) Residuals for the highecut continuum model and $\mathrm{Fe} \mathrm{K} \alpha$ feature with no absorption line included in the model. (d) Residuals for the npex model with the possible absorption line modeled by a Gaussian profile included in the model. (e) Residuals with no absorption line included in the npex model. (f) Residuals for the cutoffpl model with the possible absorption line modeled by a Gaussian profile included in the model. (g) Residuals with no absorption line included in the cutoffpl model.

inner binary is modulated at a long-term period by tidal forces of a third companion star orbiting the center of mass between it and the inner binary (Mazeh \& Shaham 1979). This period is inversely proportional to the period of the inner binary, and directly proportional to the orbital period of the third companion (see Equation (19) in Zdziarski et al. 2007). If this model is applied to IGR J16493-4348, we calculate the third period to be $11.666 \pm 0.002$ days. This period is only a factor of $\sim 1.7$ times larger than the binary orbital period measured with RXTE and Swift (Pearlman et al. 2019), which may imply an unstable orbital configuration if IGR J16493-4348 were part of a triple-star system.

The stability of a triple-star system depends on the ratio between the semimajor axis of the third companion star and the orbital separation of the components in the inner binary (Zdziarski et al. 2007; Mikkola 2008). Combining the ratio between the outer and inner periods with Kepler's third law, we calculate the ratio between the outer and inner semimajor axes

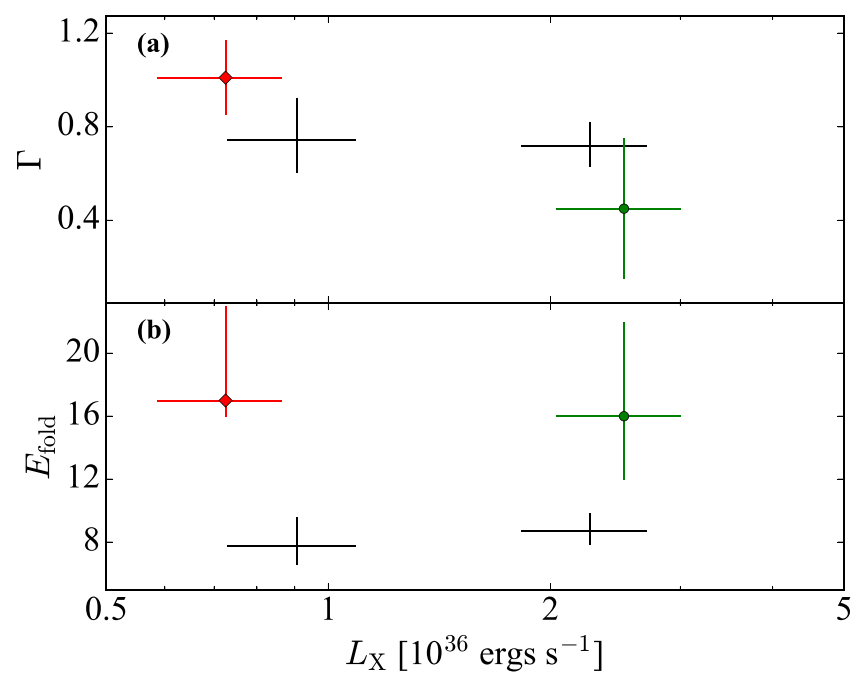

Figure 14. Evolution of the power-law (a) photon index and (b) folding energy with the 1-10 keV X-ray luminosity for the npex model. The Suzaku/Swift BAT/INTEGRAL ISGRI (D'Aì et al. 2011), Suzaku/Swift BAT/INTEGRAL ISGRI (D'Aì et al. 2011), and NuSTAR results (this work) are indicated by the green circles, red diamonds, and black crosses, respectively.

to be $\sim 1.4$. This close configuration may result in perturbations of the binary motion on timescales between the orbital period and the superorbital modulation, which were not observed with RXTE or Swift (see, e.g., Pearlman et al. 2019). It is worth noting that, depending on the mass of the third companion star, strong perturbations from a binary orbit may be detectable in pulsar timing residuals (e.g., PSR J0337+1715; Ransom et al. 2014). These perturbations were not found in the RXTE pulsar timing residuals reported in Pearlman et al. (2019), providing additional evidence against a third companion star.

\subsubsection{Precession of the Donor Star}

If a precessing donor star surrounded by an equatorially enhanced wind were the cause of the $\sim 20.06$ day period in IGR J16493-4348, long-term changes in the neutral hydrogen absorption column density may be observed (e.g., GX 304-1; Kühnel et al. 2017). We do not find any significant variations in $N_{\mathrm{H}}$ between superorbital minimum and maximum. However, we caution against ruling out a precessing equatorial wind since the absorbing material might not be along the line of sight.

\subsubsection{Corotating Interaction Regions in the Stellar Wind}

An alternative possibility is that the $\sim 20.6$ day cycle could be driven by large-scale corotating interaction regions (CIRs) in the wind of the B0.5 Ia donor star (Bozzo et al. 2017). Changes in the mass accretion rate may be partially modulated by the interaction between the neutron star and the CIRs. Phaselocked flares, possibly attributed to large-scale structures in the wind of the donor star, have been identified in the supergiant fast X-ray transient (SFXT) IGR J16479-4514 (Sidoli et al. 2013), which also shows strong superorbital modulation.

In a non-synchronous rotating binary, the angular velocities of the neutron star and the CIR would be different (see Bozzo et al. 2017 and references therein), resulting in a beat period on superorbital timescales. Bozzo et al. applied this model to IGR J16493-4348 and found that a single CIR with a period of $\sim 10.3$ days could explain the $\sim 20.06$ day superorbital period. We note the $\sim 20.6$ day modulation is persistently detected in 
the dynamic power spectrum spanning a timescale of more than $12 \mathrm{yr}$ (see Figure 2(a)). This suggests that, if CIRs are the cause of the superorbital modulation in these systems, they would have to be stable over timescales of several years (Pearlman et al. 2019).

\subsubsection{Tidal Oscillations}

Finally, we discuss the possible case that the superorbital modulation in IGR J16493-4348 is driven by a nonsynchronously rotating donor star (Moreno et al. 2005; Koenigsberger et al. 2006), which could exhibit several different periodicities due to tidal oscillations (Zahn 1977; Moreno et al. 2005). Such oscillations could produce a localized structured wind, which would drive periodic modulation in the X-ray band when accreted onto the neutron star. The period of these oscillations was calculated to be on superorbital timescales for a circular orbit. From a pulsar timing analysis, Pearlman et al. (2019) showed that the binary is likely in a nearly circular orbit, which meets the requirement of the tidal oscillation model.

\section{Summary and Conclusions}

In this paper, we have presented two NUSTAR observations of IGR J16493-4348, which coincide with the minimum and maximum of one cycle of its $\sim 20$ day superorbital modulation, and long-term observations of the superorbital period by Swift BAT. An analysis of the BAT data using the dynamic power spectra and fractional rms methods reveals strong variations in the amplitude of the superorbital modulation, but we do not observe changes in the period. The fractional rms of the $\sim 20.06$ day period closely tracks the peak power, providing additional evidence that its amplitude significantly changes with time.

Our results indicate the neutron star rotation period is consistent with that reported by Pearlman et al. (2019) at the $1 \sigma$ confidence interval. This suggests that no significant long-term neutron star rotation period derivative was detected between the RXTE and NUSTAR observations. No significant changes in the $3-50 \mathrm{keV}$ pulse profiles between the two observations are found, which suggests a similar accretion regime at superorbital minimum and maximum.

We have presented a pulse-phase-resolved spectral analysis of IGR J16493-4348 for the first time. Our results show that, while the joint NuSTAR and Swift XRT pulse-phase-averaged spectral continuum revealed no significant changes between superorbital minimum and maximum, we observe possible evidence of luminosity-dependent variability in the pulse-phase-resolved spectra. We found the spectral shape near the broad main peak of the pulse profile hardens between superorbital minimum and superorbital maximum, which is consistent with the subcritical accretion regime. It may be possible that the spectral hardness evolution seen in IGR J16493-4348 could be explained by thermal Comptonization in a collisionless shock model (e.g., Cep X-4; Vybornov et al. 2017).

We also found a weak emission line at $6.4 \mathrm{keV}$ at superorbital maximum, but it is not significant at superorbital minimum. The origin of the line is due to neutral $\mathrm{Fe}$ or $\mathrm{Fe}$ in a low ionization state, which is present in many X-ray binaries. Our pulse-phase-resolved analysis indicates that the flux of the $\mathrm{Fe} \mathrm{K} \alpha$ line does not track the pulse profile, a possible indication that the region responsible for the $\mathrm{Fe} \mathrm{K} \alpha$ emission is not close to the neutron star.
Our NUSTAR and Swift analysis shows that, while the mechanism responsible for the superorbital modulation remains elusive, we can now begin to constrain it. Mechanisms where we might expect a significant change in the neutral hydrogen column density - a precessing accretion disk, a precessing equatorially enhanced wind-are unlikely. A transient accretion disk may also be unlikely since the neutron star shows no indications of a rapid spin-up torque (Pearlman et al. 2019). A triple-star system is unlikely since the period of the third object is calculated to be $11.666 \pm 0.002$ days, which may lead to an unstable orbital configuration.

The superorbital dependence of the spectral shape in IGR J16493-4348, particularly near the broad main peak of the pulse profile, shows similarities to $2 \mathrm{~S} 0114+650$, which is the prototypical wind-fed SGXB exhibiting superorbital modulation (Farrell et al. 2006). While the spectral shape in each source hardens from superorbital minimum to maximum, an anticorrelation between photon index and X-ray luminosity is only observed in $2 \mathrm{~S} 0114+650$. The behavior of spectral hardness in both sources, however, may suggest that the superorbital mechanism is linked to a variable accretion rate. Superorbital mechanisms that explain the variable accretion rate such as tidal oscillations or large structures in the donor star wind remain possible.

To further understand the mechanism responsible for the $\sim 20.06$ day superorbital cycle in IGR J16493-4348, additional multi-wavelength observations are required. The study would benefit from optical/near-infrared photometry, which may confirm or preclude variations in the donor star or its wind as the driving mechanism of the superorbital modulation.

We thank the anonymous referee for useful comments. We also thank Drs. Patricia Boyd, Sebastian Falkner, Illeyk El Mellah, and Enrico Bozzo for useful discussions, and the NUSTAR Operations, Software and Calibration teams for scheduling and the execution of these observations. A.B.P. acknowledges support by the Department of Defense (DoD) through the National Defense Science and Engineering Graduate (NDSEG) Fellowship Program and by the National Science Foundation (NSF) Graduate Research Fellowship under grant No. DGE-1144469. This research has made use of the XRT Data Analysis Software (XRTDAS) developed under the responsibility of the ASI Science Data Center (ASDC), Italy and the NuSTAR Data Analysis Software (NuSTARDAS) jointly developed by the ASI Science Science Data Center (ASDC, Italy) and the California Institute of Technology. We thank NASA's 14ADAP14-0167 grant and NuSTAR Guest Observer grant 14NUSTAR14-0007 for support.

\section{ORCID iDs}

Joel B. Coley (iD https://orcid.org/0000-0001-7532-8359

Robin H. D. Corbet (i) https://orcid.org/0000-00023396-651X

Felix Fürst (ib https://orcid.org/0000-0003-0388-0560

Hans A. Krimm (i) https://orcid.org/0000-0003-4348-6058

Aaron B. Pearlman (iD https://orcid.org/0000-0002-8912-0732

Katja Pottschmidt (iD https://orcid.org/0000-0002-4656-6881

\section{References}

Bachetti, M., Harrison, F. A., Walton, D. J., et al. 2014, Natur, 514, 202 Ballhausen, R., Pottschmidt, K., Fürst, F., et al. 2017, A\&A, 608, A105 Barthelmy, S. D., Barbier, L. M., Cummings, J. R., et al. 2005, SSRv, 120, 143 
Becker, P. A., Klochkov, D., Schönherr, G., et al. 2012, A\&A, 544, A123 Bird, A. J., Barlow, E. J., Bassani, L., et al. 2004, ApJL, 607, L33 Bondi, H., \& Hoyle, F. 1944, MNRAS, 104, 273

Bozzo, E., Oskinova, L., Lobel, A., \& Hamann, W.-R. 2017, A\&A, 606, L10 Brightman, M., Harrison, F. A., Bachetti, M., et al. 2019, ApJ, 873, 115 Brumback, M. C., Hickox, R. C., Fürst, F. S., et al. 2018, ApJ, 852, 132

Burrows, D. N., Hill, J. E., Nousek, J. A., et al. 2005, SSRv, 120, 165

Capalbi, M., Perri, M., Saija, B., Tamburelli, F., \& Angelini, L. 2005, The Swift XRT Data Reduction Guide, Technical Report 1.2, http://swift.gsfc. nasa.gov/analysis/xrt_swguide_v1_2.pdf

Chaty, S. 2011, in ASP Conf. Ser. 29, Evolution of Compact Binaries, ed. L. Schmidtobreick, M. R. Schreiber, \& C. Tappert (San Francisco, CA: ASP), 447

Chou, Y., \& Grindlay, J. E. 2001, ApJ, 563, 934

Clarkson, W. I., Charles, P. A., Coe, M. J., et al. 2003, MNRAS, 339, 447

Coburn, W., Heindl, W. A., Rothschild, R. E., et al. 2002, ApJ, 580, 394

Corbet, R., Markwardt, C., Barbier, L., et al. 2007, PThPS, 169, 200

Corbet, R. H. D., Barthelmy, S. D., Baumgartner, W. H., et al. 2010a, ATel, 2599, 1

Corbet, R. H. D., Coley, J. B., \& Krimm, H. A. 2017, ApJ, 846, 161

Corbet, R. H. D., Coley, J. B., Krimm, H. A., \& Pottschmidt, K. 2018, ATel, 11918,1

Corbet, R. H. D., \& Krimm, H. A. 2013, ApJ, 778, 45

Corbet, R. H. D., Pearlman, A. B., \& Pottschmidt, K. 2010b, ATel, 2766, 1

Cusumano, G., La Parola, V., Segreto, A., et al. 2010, A\&A, 510, A48

D’Aì, A., Cusumano, G., La Parola, V., et al. 2011, A\&A, 532, A73

Dickey, J. M., \& Lockman, F. J. 1990, ARA\&A, 28, 215

El Mellah, I., Sander, A. A. C., Sundqvist, J. O., \& Keppens, R. 2019, A\&A, 622, A189

Falkner, S., Schwarm, F.-W., Dauser, T., et al. 2019, A\&A, submitted

Farrell, S. A., Sood, R. K., \& O'Neill, P. M. 2006, MNRAS, 367, 1457

Farrell, S. A., Sood, R. K., O’Neill, P. M., \& Dieters, S. 2008, MNRAS, 389,608

Fürst, F., Walton, D. J., Heida, M., et al. 2018, A\&A, 616, A186

Fürst, F., Walton, D. J., Stern, D., et al. 2017, ApJ, 834, 77

Grebenev, S. A., Bird, A. J., Molkov, S. V., et al. 2005, ATel, 457, 1

Harrison, F. A., Craig, W. W., Christensen, F. E., et al. 2013, ApJ, 770, 103

Hill, A. B., Dean, A. J., Landi, R., et al. 2008, MNRAS, 385, 423

Hill, J. E., Burrows, D. N., Nousek, J. A., et al. 2004, Proc. SPIE, 5165, 217

Horne, J. H., \& Baliunas, S. L. 1986, ApJ, 302, 757

Hu, C.-P., Chou, Y., Ng, C.-Y., Chun-Che Lin, L., \& Chien-Chang Yen, D. 2017, ApJ, 844, 16

Jenke, P. A., Finger, M. H., Wilson-Hodge, C. A., \& Camero-Arranz, A. 2012, ApJ, 759, 124

Kalberla, P. M. W., Burton, W. B., Hartmann, D., et al. 2005, A\&A, 440, 775

Klochkov, D., Staubert, R., Santangelo, A., Rothschild, R. E., \& Ferrigno, C. 2011, A\&A, 532, A126

Klochkov, D. K., Shakura, N. I., Postnov, K. A., et al. 2006, AstL, 32, 804

Koen, C. 1990, ApJ, 348, 700

Koenigsberger, G., Georgiev, L., Moreno, E., et al. 2006, A\&A, 458, 513
Kraus, U., Rebetzky, A., Herold, H., et al. 1989, in ESA Special Publications, Two Topics in X-Ray Astronomy, Vol. 1, ed. J. Hunt \& B. Battrick (Noordwijk: ESA), 433

Krimm, H. A., Holland, S. T., Corbet, R. H. D., et al. 2013, ApJS, 209, 14

Kühnel, M., Falkner, S., Grossberger, C., et al. 2016, AcPol, 56, 41

Kühnel, M., Rothschild, R. E., Okazaki, A. T., et al. 2017, MNRAS, 471, 1553

Kuiper, L., Jonker, P., Hermsen, W., \& O’Brien, K. 2005, ATel, 654, 1

Leahy, D. A. 1987, A\&A, 180, 275

Makishima, K., Mihara, T., Nagase, F., \& Tanaka, Y. 1999, ApJ, 525, 978

Markwardt, C. B., Swank, J. H., \& Smith, E. 2005, ATel, 465, 1

Mazeh, T., \& Shaham, J. 1979, A\&A, 77, 145

Meszaros, P., \& Nagel, W. 1985, ApJ, 299, 138

Mikkola, S. 2008, in Multiple Stars Across the H-R Diagram, ed. S. Hubrig, M. Petr-Gotzens, \& A. Tokovinin (Berlin: Springer), 11

Molkov, S. V., Lutovinov, A. A., \& Falanga, M. 2015, AstL, 41, 562

Moreno, E., Koenigsberger, G., \& Toledano, O. 2005, A\&A, 437, 641

Morris, D. C., Smith, R. K., Markwardt, C. B., et al. 2009, ApJ, 699, 892

Nespoli, E., Fabregat, J., \& Mennickent, R. E. 2010, A\&A, 516, A106

Ogilvie, G. I., \& Dubus, G. 2001, MNRAS, 320, 485

Pearlman, A. B., Coley, J. B., Corbet, R. H. D., \& Pottschmidt, K. 2019, ApJ, 873,86

Pike, S. N., Harrison, F. A., Bachetti, M., et al. 2019, ApJ, 875, 144

Postnov, K., Shakura, N., Staubert, R., et al. 2013, MNRAS, 435, 1147

Postnov, K. A., Gornostaev, M. I., Klochkov, D., et al. 2015, MNRAS, 452, 1601

Pringle, J. E. 1996, MNRAS, 281, 357

Protassov, R., van Dyk, D. A., Connors, A., Kashyap, V. L., \& Siemiginowska, A. 2002, ApJ, 571, 545

Ramsay, G., Zane, S., Jimenez-Garate, M. A., den Herder, J.-W., \& Hailey, C. J. 2002, MNRAS, 337, 1185

Ransom, S. M., Stairs, I. H., Archibald, A. M., et al. 2014, Natur, 505, 520

Reig, P., Chakrabarty, D., Coe, M. J., et al. 1996, A\&A, 311, 879

Scargle, J. D. 1982, ApJ, 263, 835

Schwarm, F.-W., Ballhausen, R., Falkner, S., et al. 2017, A\&A, 601, A99

Scott, D. M., Leahy, D. A., \& Wilson, R. B. 2000, ApJ, 539, 392

Sidoli, L., Esposito, P., Sguera, V., et al. 2013, MNRAS, 429, 2763

Taani, A., Karino, S., Song, L., et al. 2019, RAA, 19, 012

Taani, A., Karino, S., Song, L., Zhang, C., \& Chaty, S. 2018, arXiv:1808.05345 Tanaka, Y. 1986, LNP, 255, 198

Trowbridge, S., Nowak, M. A., \& Wilms, J. 2007, ApJ, 670, 624

Tueller, J., Baumgartner, W. H., Markwardt, C. B., et al. 2010, ApJS, 186, 378

Vaughan, S., Edelson, R., Warwick, R. S., \& Uttley, P. 2003, MNRAS, 345, 1271

Verner, D. A., Ferland, G. J., Korista, K. T., \& Yakovlev, D. G. 1996, ApJ, 465,487

Vybornov, V., Klochkov, D., Gornostaev, M., et al. 2017, A\&A, 601, A126

Walton, D. J., Fürst, F., Bachetti, M., et al. 2016, ApJL, 827, L13

White, N. E., Swank, J. H., \& Holt, S. S. 1983, ApJ, 270, 711

Wilms, J., Allen, A., \& McCray, R. 2000, ApJ, 542, 914

Winkler, C., Courvoisier, T. J.-L., Di Cocco, G., et al. 2003, A\&A, 411, L1

Zahn, J.-P. 1977, A\&A, 57, 383

Zdziarski, A. A., Wen, L., \& Gierliński, M. 2007, MNRAS, 377, 1006 